\title{
OPEN An integrated set-up for ex vivo characterisation of biaxial murine artery biomechanics under pulsatile conditions
}

\author{
Myrthe M. van der Bruggen ${ }^{1}$, Koen D. Reesink ${ }^{1}$, Paul J. M. Spronck ${ }^{2}$, Nicole Bitsch ${ }^{3}$, \\ Jeroen Hameleers ${ }^{1}$, Remco T. A. Megens ${ }^{1,4}$, Casper G. Schalkwijk ${ }^{5}$, Tammo Delhaas ${ }^{1} \&$ \\ Bart Spronck ${ }^{1,6 \bowtie}$
}

Ex vivo characterisation of arterial biomechanics enables detailed discrimination of the various cellular and extracellular contributions to arterial stiffness. However, ex vivo biomechanical studies are commonly performed under quasi-static conditions, whereas dynamic biomechanical behaviour (as relevant in vivo) may differ substantially. Hence, we aim to (1) develop an integrated set-up for quasi-static and dynamic biaxial biomechanical testing, (2) quantify set-up reproducibility, and (3) illustrate the differences in measured arterial stiffness between quasi-static and dynamic conditions. Twenty-two mouse carotid arteries were mounted between glass micropipettes and kept fully vasodilated. While recording pressure, axial force $(F)$, and inner diameter, arteries were exposed to (1) quasi-static pressure inflation from 0 to $200 \mathrm{mmHg}$ (2) $300 \mathrm{bpm}$ dynamic pressure inflation (peaking at $80 / 120 / 160 \mathrm{mmHg}$ ); and (3) axial stretch $\left(\lambda_{z}\right)$ variation at constant pressures of 10/60/100/140/200 mm Hg. Measurements were performed in duplicate. Single-point pulse wave velocities (PWV; Bramwell-Hill) and axial stiffness coefficients $\left(c_{\mathrm{ax}}=\mathrm{d} F / \mathrm{d} \lambda_{z}\right)$ were calculated at the in vivo value of $\lambda_{z}$. Within-subject coefficients of variation were $\sim 20 \%$. Dynamic PWVs were consistently higher than quasi-static PWVs $(p<0.001) ; c_{\mathrm{ax}}$ increased with increasing pressure. We demonstrated the feasibility of ex vivo biomechanical characterisation of biaxially-loaded murine carotid arteries under pulsatile conditions, and quantified reproducibility allowing for well-powered future study design.

Large artery stiffness is a strong and independent predictor for cardiovascular events as well as all-cause mortality in the general population ${ }^{1-5}$. Therefore, treatment of arterial stiffness is considered to be a good target in prevention of cardiovascular disease ${ }^{6-9}$. Clinical arterial stiffness measurements, however, do not yield direct insight in the underlying biomechanics of the arterial wall. To enable more detailed assessment of biomechanical properties, ex vivo experimental studies are useful. Ex vivo characterisation allows more tightly controlled hemodynamic and mechanical conditions, enabling discrimination of e.g. cellular and extracellular processes in vascular remodelling ${ }^{10}$. Importantly, in contrast to in vivo measurements, ex vivo studies allow biomechanical characterisation beyond the physiological pressure range. Moreover, biaxial data on pressure, diameter, length, and axial force of the artery can be recorded simultaneously during ex vivo experiments. Integration of the data using a computational model of arterial wall mechanics is useful to obtain quantitative insight into the constitutive properties of arteries $^{11,12}$.

Whereas most ex vivo experiments on arteries are performed under quasi-static conditions (i.e. slowly increasing pressure), cyclic stretch at a physiological rate emerged as a major determinant of vascular function and mechanical homeostasis ${ }^{7,13-16}$. Previous studies imply that arterial biomechanical behaviour may substantially

\footnotetext{
${ }^{1}$ Department of Biomedical Engineering, CARIM School for Cardiovascular Diseases, Maastricht University, Universiteitssingel 50, Room 3.359, 6229ER Maastricht, The Netherlands. 'Innovatest Europe BV, Maastricht, The Netherlands. ${ }^{3}$ Muroidean Facility, CARIM School for Cardiovascular Diseases, Maastricht University, Maastricht, The Netherlands. ${ }^{4}$ Institute for Cardiovascular Prevention, Ludwig-Maximilians-Universität, Munich, Germany. ${ }^{5}$ Department of Internal Medicine, CARIM School for Cardiovascular Diseases, Maastricht University Medical Centre+, Maastricht, The Netherlands. ${ }^{6}$ Department of Biomedical Engineering, School of Engineering \& Applied Science, Yale University, New Haven, CT, USA. ${ }^{\circledR}$ email: b.spronck@maastrichtuniversity.nl
} 


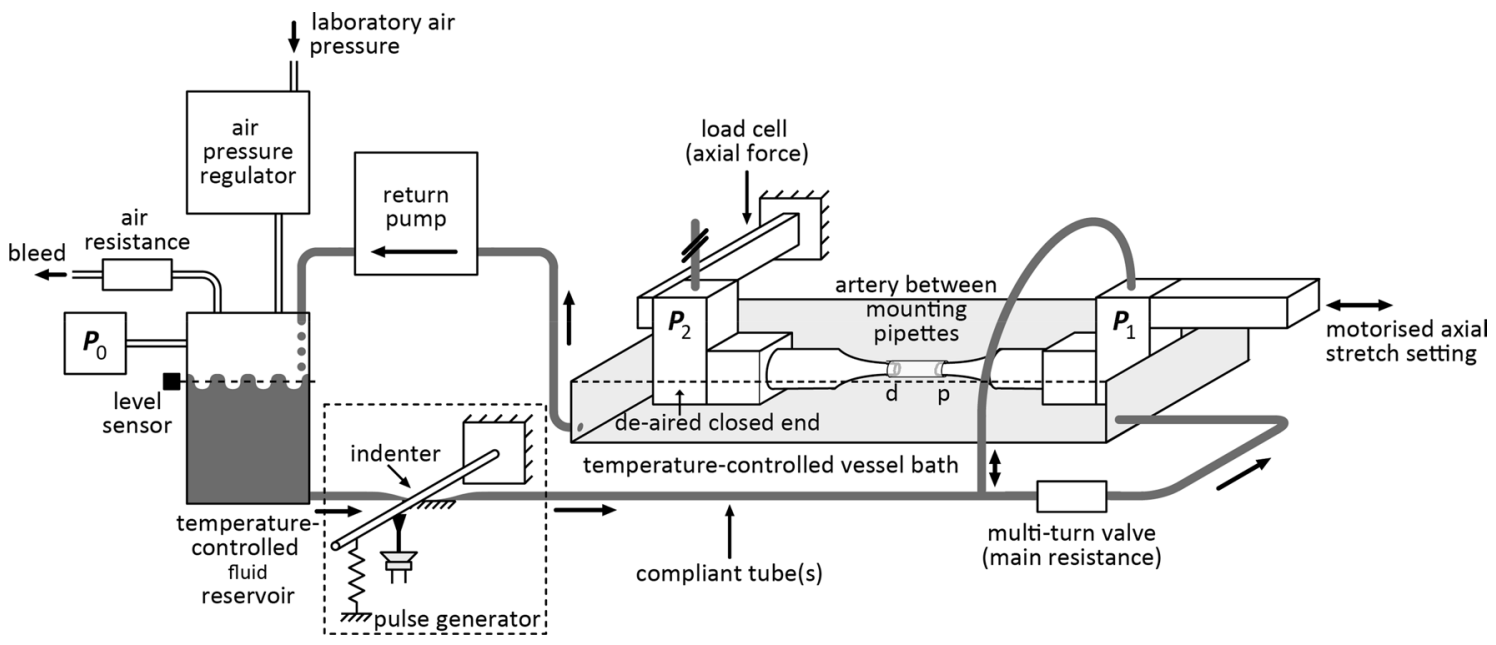

Figure 1. Set-up specialised for biaxial biomechanical assessment of murine arteries under pulsatile conditions. $\mathrm{p}$ and $\mathrm{d}$, proximal and distal pipettes for mounting the artery. $P_{0}, P_{1}$, and $P_{2}$ are sensors registering reservoir and proximal and distal pipette pressures, respectively. Note that sensor $P_{2}$ is at a de-aired closed end. For detailed description, see main text.

differ under dynamic and static conditions ${ }^{16-20}$. A number of studies have performed ex vivo dynamic assessment of large artery stiffness and distensibility, in e.g. rat $^{21}$ and pig $^{22}$. To our knowledge, the first study subjecting biaxially loaded murine carotid arteries to pulsatile pressure was by Gleason et al. ${ }^{10}$. However, pressure waves in their set-up were sinusoidal and the sites of pressure measurement were distant from the mounting pipettes. Furthermore, because of the pipette impedances and nonzero flow through them, a pressure measured through a narrow pipette will exhibit a (frequency-dependent) phase difference with measured diameter, which potentially hampers correct identification of viscous and elastic contributions to artery wall stress-strain behaviour ${ }^{23}$.

A potential solution to this problem is to employ a wire myography-based technique, as performed by Leloup et al. ${ }^{7}$. Although using such technique, circumferential force and displacement can be directly measured, axial stretch cannot be manipulated, prohibiting the study of the axial biomechanical behaviour. Furthermore, because of the lack of axial constraint, also during circumferential measurements, vessels will not be held at an in vivo relevant axial stretch ( $\lambda_{z}$; in the order of $\sim 1.7$ for carotid arteries). Instead, in wire myography, the axial stretch during measurement will typically be smaller than unity $\left(\lambda_{z}<1\right.$, i.e. the vessels are axially compressed instead of stretched). This non-physiological axial stretching state has important functional consequences: e.g. it influences sensitivity to vasoactive substances ${ }^{24}$, but also directly influences measured circumferential properties due to axial-circumferential coupling ${ }^{25}$.

In the present study, we describe and characterise a set-up for integrated biomechanical characterisation of biaxially loaded passive (i.e. without vascular smooth muscle cell (VSMC) contribution) murine carotid arteries under pulsatile as well as quasi-static conditions, closely mimicking in vivo conditions. Specifically, we aim to (1) present the details of our set-up, (2) quantify set-up reproducibility, and (3) illustrate the difference in measured arterial stiffness between testing under quasi-static and dynamic conditions.

The set-up presented herein has a pipette and pressure recording configuration that avoids the aforementioned frequency-dependent phase errors. By closing and de-airing the outflow end of the system, there was no flow across the distal pipette (Fig. 1). The distal pipette acts as an extension of the pressure sensor, ultimately eliminating the problem of pipette impedance. Vessels with a typical loaded outer diameter of $0.7 \mathrm{~mm}$ can be tested, making our set-up highly suitable for biomechanical characterisation of (large) arteries of the mouse, an animal model abundant in the field. Inflation experiments under quasi-static as well as dynamic (i.e. close to in vivo) conditions at pressures ranging from 0 to $200 \mathrm{mmHg}$ and a pulse frequency of $5 \mathrm{~Hz}(300 \mathrm{bpm})$ are performed with the vessel at or around its in vivo value of axial stretch, yielding relevant loading conditions. In addition, axial extension measurements at fixed pressure levels allow for quantification of axial mechanical behaviour.

\section{Results}

Choice of pressure measurement location to represent intravascular pressure. The pipette configuration we used (Fig. 1) is essential for obtaining dynamic pressure-diameter curves with negligible phaseand amplitude errors. We chose to use one pipette (proximal (p) in Fig. 1) to inflate/deflate the artery, and the other pipette (distal (d)) to obtain a representative intravascular pressure measurement at sensor $P_{2}$. Lumpedparameter modelling simulations indeed showed no phase error between intravascular pressure $\left(P_{\text {vessel }}\right)$ and $P_{2}$ for the reference situation (at an $R C$ time of $5 \mathrm{~ms}$, typical for our set-up and a control carotid artery), whereas $P_{1}$ showed a frequency-dependent phase error of 2 degrees at $1 \mathrm{~Hz}$ up to 18 degrees at $10 \mathrm{~Hz}$ (continuous lines; Fig. 2). Correspondingly, the amplitude error remains zero for $P_{2}$, whereas for $P_{1}$ e.g. a $5 \%$ overestimation is attained at $10 \mathrm{~Hz}$. Importantly, with a threefold increase in vessel compliance (dashed lines; $R C$ time $=15 \mathrm{~ms}$ ) the phase and amplitude errors for $P_{1}$ increase, whereas those for $P_{2}$ remain unaffected (Fig. 2). It should be noted that because our set-up generates physiological pressure waveforms (cf. Fig. 3 ) the relevant bandwidth is about 


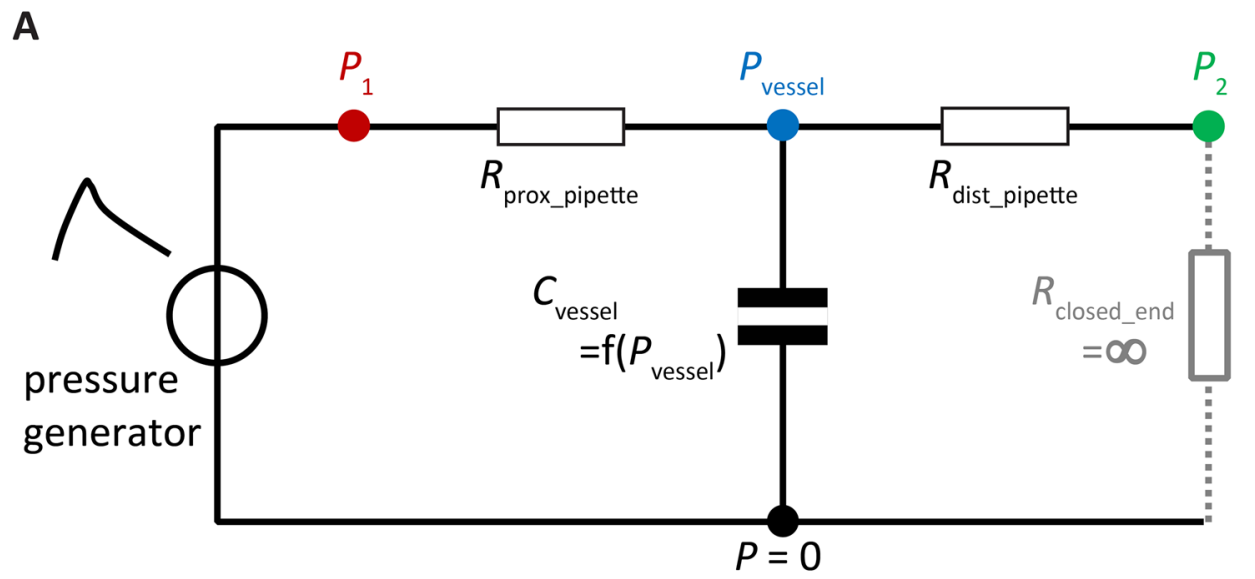

B

extramural pressure

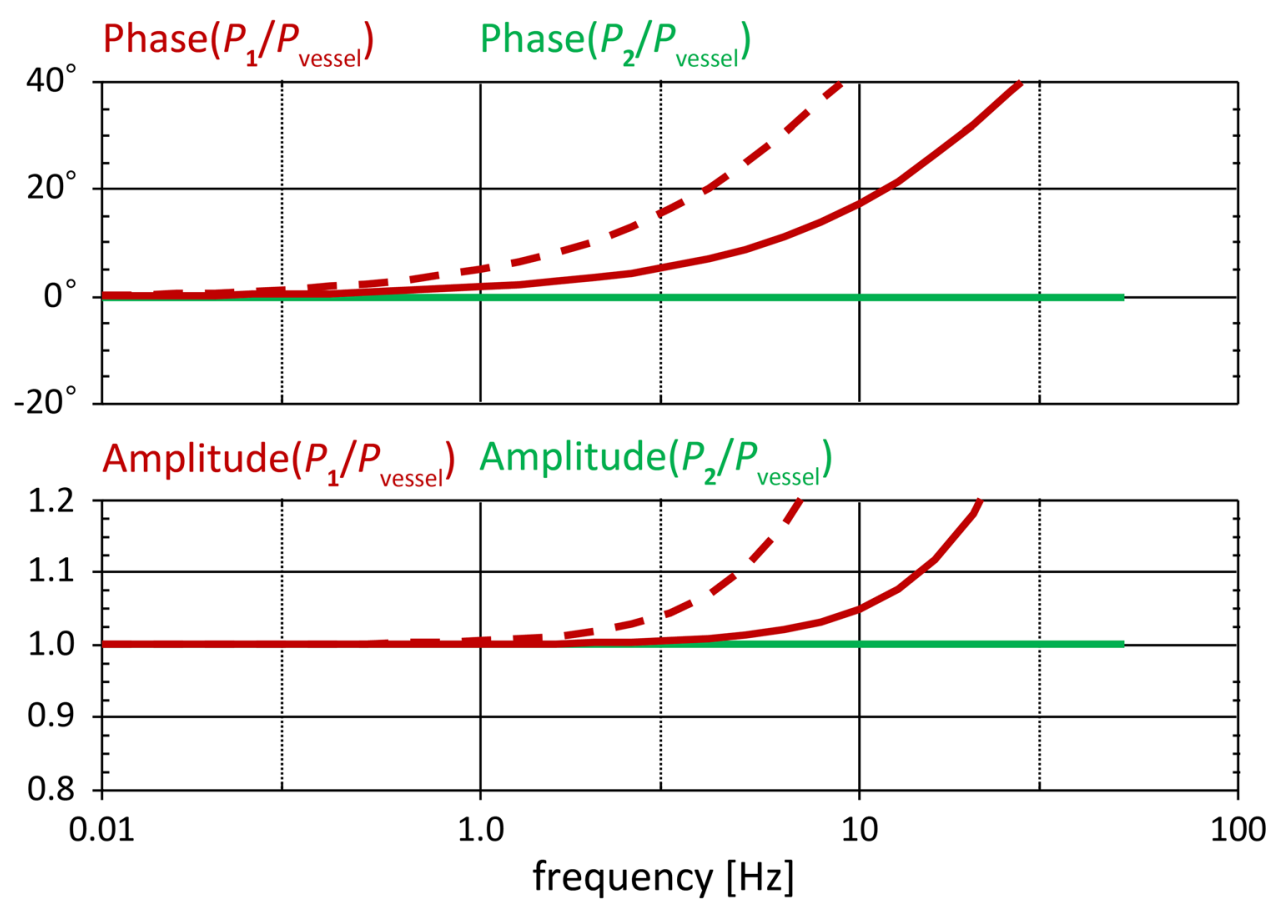

Figure 2. (A) Schematic model of the pipette configuration and pressure recording positions. $P_{1}$ indicates the proximal pressure sensor; $P_{2}$ indicates the distal pressure sensor; and $P_{\text {vessel }}$ indicates the true pressure inside the vessel under test. $R_{\text {closed end }}$ models the stopcock distal to sensor $P_{2}$ which during measurements is closed to prevent any flow through the distal pipette (modelled by $\left.R_{\text {dist_pipette }}\right)$. We used the " $C_{\text {vessel }}=\mathrm{f}\left(P_{\text {vessel }}\right.$ )" notation to indicate that $C_{\text {vessel }}$ is strongly dependent on transmural pressure (due to nonlinear arterial elasticity). (B) Phase (top) and amplitude (bottom) bode diagrams, showing amplitude relationships of $P_{2}$ and $P_{1}$ to $P_{\text {vessel, }}$, as a function of frequency. A phase of zero degrees indicates no phase error; an amplitude of 1.0 indicates no amplitude error. Continuous lines indicate the situation for an $R C$-time (defined as $R_{\text {prox_pipette }} \cdot C_{\text {vessel }}$ ) of about $5 \mathrm{~ms}$ and the dashed lines for a tripled $R C$-time (i.e. $15 \mathrm{~ms}$, with $C_{\text {vessel }}$ assumed 3 times larger and $R_{\text {prox_pipette }}$ kept constant). $P_{1}$ shows considerable, frequency-dependent errors, whereas $P_{2}$ shows flat curves with no errors in the relevant frequency range (i.e. $5 \mathrm{~Hz}$ cycle times 10 harmonics to represent the waveform requires at least $50 \mathrm{~Hz}$ ). Taken together, pressure as recorded at $P_{2}$ is representative of $P_{\text {vessel. }}$. In contrast, $P_{1}$ shows a large, frequency and compliance-dependent difference (error) from $P_{\text {vessel- }}$.

$50 \mathrm{~Hz}$ (i.e. 10 harmonics at a base frequency of $5 \mathrm{~Hz}$ ). Hence, if $P_{1}$ would be used to represent $P_{\text {vessel, }}$, a substantial overestimation and phase error would occur at frequencies within this relevant bandwidth. When using $P_{2}$ to represent $P_{\text {vessel }}$, these problems are negligible. 
A
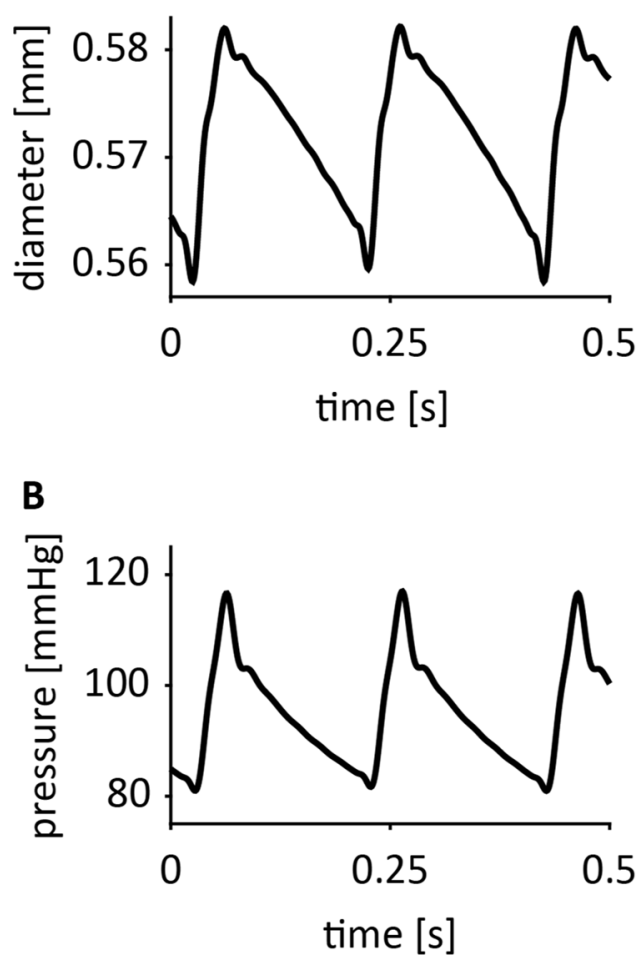

C

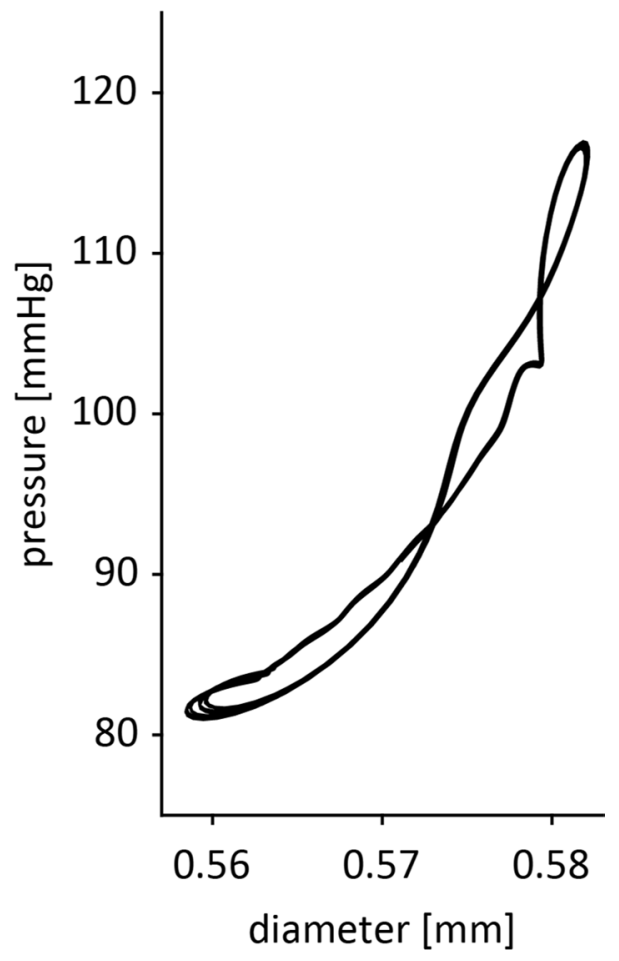

Figure 3. Representative examples of inner diameter (A) and pressure (B) tracings, and the resulting pressurediameter plot (C), exposing considerable curvilinearity but rather limited loop area.

Set-up reproducibility. Within-subject coefficients of variation (CVs) for quasi-static and dynamic pulse wave velocity (PWV) and axial stiffness coefficient were approximately $20 \%$. Detailed reproducibility statistics are presented in Table 1.

Quasi-static inflation. Axial stretch significantly influenced quasi-static PWV measured at pressure range $120 / 80 \mathrm{mmHg}\left(\chi^{2}(2)=28.3, p<0.001\right)$. Dunn-Bonferroni post hoc testing revealed PWV at $\lambda_{z}=0.95 \lambda_{z, i v}(4.6$ $[3.7-5.0] \mathrm{m} / \mathrm{s})$ to be significantly lower than at $\lambda_{z}=\lambda_{z, i v}(4.9[4.2-5.5] \mathrm{m} / \mathrm{s}, p<0.001)$ and $\lambda_{z}=1.05 \lambda_{z, i v}(5.2$ [4.4-5.7] $\mathrm{m} / \mathrm{s}, p<0.001$; Fig. $4 \mathrm{~A} / \mathrm{B})$, with $\lambda_{z \text {,iv }}$ indicating the estimated axial stretch that the artery was at in vivo (see "Methods" section).

Similar results were found for quasi-static distensibility coefficient (DC) measured at the pressure range of $120 / 80 \mathrm{mmHg}\left(\chi^{2}(2)=25.9, \mathrm{p}<0.001\right)$. Post hoc testing revealed a significant difference between $\lambda_{z}=0.95 \lambda_{z, i v}$ and $\lambda_{z}=\lambda_{z, i v}\left(49.4\right.$ [40.3-69.8] vs. 47.3 [33.6-55.6], $\left.\mathrm{MPa}^{-1}, \mathrm{p}=0.001\right)$. This significant difference was also found between $\lambda_{z}=0.95 \lambda_{z, i v}$ and $\lambda_{z}=1.05 \lambda_{z, i v}$ (36.8 [29.8-50.7], $\mathrm{MPa}^{-1}, \mathrm{p}<0.001$; Fig. 4C).

Finally, these results were confirmed in quasi-static compliance coefficient (CC) outcomes as well $\left(\chi^{2}(2)=34.3\right.$ $p<0.001)$. Again, CC at $\lambda_{z}=0.95 \lambda_{z, i v}(1.54[1.32-2.35] \mu \mathrm{m} / \mathrm{mmHg})$ to be significantly higher compared to $\lambda_{z}=\lambda_{z, i v}(1.48[1.14-1.86] \mu \mathrm{m} / \mathrm{mmHg}, p=0.005)$ and $\lambda_{z}=1.05 \lambda_{z, i v}(1.09[0.91-1.61] \mu \mathrm{m} / \mathrm{mmHg}, p<0.001$; Fig. 4). $\lambda_{z}=\lambda_{z, i v}$ and $\lambda_{z}=1.05 \lambda_{z, i v}$ also significantly differed in this analysis ( $p=0.022$; Fig. $\left.4 \mathrm{D}\right)$.

Dynamic (pulsatile) inflation. Dynamic PWV measured at $5 \mathrm{~Hz}(300 \mathrm{bpm})$ was consistently higher than quasi-static PWV for $80 \mathrm{mmHg}(2.3$ [2.2-2.5] vs. 2.1 [1.9-2.2] m/s, $p<0.001), 120 \mathrm{mmHg}(5.9$ [5.4-6.4] vs. 4.9 [4.2-5.5] m/s, $p<0.001)$, and $160 \mathrm{mmHg}(14.4$ [11.6-15.5] vs. 10.8 [9.9-12.3] m/s, $p=0.001$; Fig. 5A,B). As expected, PWV showed an increase with increasing transmural pressure for both the dynamic and quasi-static measurements $\left(\chi^{2}(2)=40.1, p<0.001 ; \chi^{2}(2)=42.0, p<0.001\right)$.

In line with PWV results, DC was higher in quasi-static measurements compared to dynamic measurements for $80 \mathrm{mmHg}\left(220.2\right.$ [197.9-251.9] vs. 178.9 [149.0-199.6] $\left.\mathrm{MPa}^{-1}, p<0.001\right), 120 \mathrm{mmHg}(47.3$ [33.6-55.6] vs. 28.1 [24.3-35.5] $\left.\mathrm{MPa}^{-1}, p<0.001\right)$, and $160 \mathrm{mmHg}\left(8.3\right.$ [6.4-10.5] vs. 5.0 [3.9-7.3] $\mathrm{MPa}^{-1}, p=0.001$; (Fig. 5C)). DC showed a decrease with increasing transmural pressure for both the dynamic and quasi-static measurements $\left(\chi^{2}(2)=40.1, p<0.001 ; \chi^{2}(2)=42.0, p<0.001\right)$.

As expected, $\mathrm{CC}$ was also higher in quasi-static compared to dynamic measurements for $80 \mathrm{mmHg}(4.97$ [4.69-5.32] vs. 4.01 [3.58-4.32], $\mu \mathrm{m} / \mathrm{mmHg}, p=0.002), 120 \mathrm{mmHg}(1.48$ [1.14-1.86] vs. 1.02 [0.87-1.20], $\mu \mathrm{m} /$ $\mathrm{mmHg}, p<0.001)$, and $160 \mathrm{mmHg}(0.30$ [0.25-0.40] vs. 0.20 [0.16-0.28], $\mu \mathrm{m} / \mathrm{mmHg}, p=0.004$ (Fig. 5D)). CC decreased with increasing transmural pressure for both the dynamic and quasi-static measurements $\left(\chi^{2}(2)=40.1\right.$, $\left.p<0.001 ; \chi^{2}(2)=42.0, p<0.001\right)$. 


\begin{tabular}{|c|c|c|c|c|c|c|c|}
\hline & \multirow[b]{2}{*}{$n_{\text {sample }}$} & \multirow[b]{2}{*}{ Mean } & \multicolumn{5}{|c|}{ Standard deviation (SD) and coefficient of variation (CV) } \\
\hline & & & & Within-subject & Between-subject & $\begin{array}{l}\text { Composite } \\
\left(n_{\text {rep }}=1\right)\end{array}$ & $\begin{array}{l}\text { Composite } \\
\left(n_{\text {rep }}=2\right)\end{array}$ \\
\hline \multicolumn{8}{|c|}{ Dynamic inflation pulse wave velocity (PWV) } \\
\hline \multirow{2}{*}{$80 \mathrm{mmHg}$} & \multirow{2}{*}{19} & \multirow{2}{*}{$\begin{array}{l}2.43(2.38-2.49) \\
\mathrm{m} / \mathrm{s}\end{array}$} & SD & $\begin{array}{l}0.43(0.30-0.50) \\
\mathrm{m} / \mathrm{s}\end{array}$ & $\begin{array}{l}0.17(0.09-0.20) \\
\mathrm{m} / \mathrm{s}\end{array}$ & $\begin{array}{l}0.46(0.35-0.53) \\
\mathrm{m} / \mathrm{s}\end{array}$ & $\begin{array}{l}0.35(0.28-0.38) \\
\mathrm{m} / \mathrm{s}\end{array}$ \\
\hline & & & $\mathrm{CV}$ & $17.5(12.5-20.4) \%$ & $7.1(3.7-8.3) \%$ & $18.9(14.4-21.2) \%$ & $14.3(11.6-15.5) \%$ \\
\hline \multirow[t]{2}{*}{$120 \mathrm{mmHg}$} & \multirow[t]{2}{*}{19} & \multirow{2}{*}{$\begin{array}{l}6.00(5.83-6.16) \\
\mathrm{m} / \mathrm{s}\end{array}$} & SD & $\begin{array}{l}1.25(1.11-1.37) \\
\mathrm{m} / \mathrm{s}\end{array}$ & $\begin{array}{l}0.65(0.07-0.83) \\
\mathrm{m} / \mathrm{s}\end{array}$ & $\begin{array}{l}1.41(1.28-1.48) \\
\mathrm{m} / \mathrm{s}\end{array}$ & $\begin{array}{l}1.10(0.93-1.18) \\
\mathrm{m} / \mathrm{s}\end{array}$ \\
\hline & & & $\mathrm{CV}$ & $20.9(18.4-22.9) \%$ & $10.8(1.1-13.9) \%$ & $23.5(21.3-24.8) \%$ & $18.3(15.4-19.7) \%$ \\
\hline \multirow{2}{*}{$160 \mathrm{mmHg}$} & \multirow[t]{2}{*}{18} & \multirow{2}{*}{$\begin{array}{l}13.49(13.12-13.86) \\
\mathrm{m} / \mathrm{s}\end{array}$} & SD & $\begin{array}{l}2.28(2.10-2.44) \\
\mathrm{m} / \mathrm{s}\end{array}$ & $\begin{array}{l}1.75(0.92-2.06) \\
\mathrm{m} / \mathrm{s}\end{array}$ & $\begin{array}{l}2.87(2.45-3.06) \\
\mathrm{m} / \mathrm{s}\end{array}$ & $\begin{array}{l}2.38(1.83-2.59) \\
\mathrm{m} / \mathrm{s}\end{array}$ \\
\hline & & & $\mathrm{CV}$ & $16.9(15.4-18.4) \%$ & $13.0(6.2-15.4) \%$ & $21.3(17.9-23.4) \%$ & $17.6(13.2-20.0) \%$ \\
\hline \multicolumn{8}{|c|}{ Quasi-static inflation pulse wave velocity (PWV) } \\
\hline \multirow{2}{*}{$80 \mathrm{mmHg}$} & \multirow[t]{2}{*}{18} & \multirow{2}{*}{$\begin{array}{l}2.15(2.10-2.20) \\
\mathrm{m} / \mathrm{s}\end{array}$} & SD & $\begin{array}{l}0.37(0.23-0.45) \\
\mathrm{m} / \mathrm{s}\end{array}$ & $\begin{array}{l}0.14(0.05-0.18) \\
\mathrm{m} / \mathrm{s}\end{array}$ & $\begin{array}{l}0.40(0.24-0.49) \\
\mathrm{m} / \mathrm{s}\end{array}$ & $\begin{array}{l}0.30(0.18-0.36) \\
\mathrm{m} / \mathrm{s}\end{array}$ \\
\hline & & & $\mathrm{CV}$ & $17.3(11.0-20.5) \%$ & $6.7(2.2-8.2) \%$ & $18.6(11.6-21.8) \%$ & $14.0(8.6-15.9) \%$ \\
\hline \multirow[t]{2}{*}{$120 \mathrm{mmHg}$} & \multirow[t]{2}{*}{18} & \multirow{2}{*}{$\begin{array}{l}4.93(4.81-5.05) \\
\mathrm{m} / \mathrm{s}\end{array}$} & SD & $\begin{array}{l}1.19(0.90-1.37) \\
\mathrm{m} / \mathrm{s}\end{array}$ & $\begin{array}{l}\mathrm{N} / \mathrm{A}^{*}(\mathrm{~N} / \mathrm{A}-0.37) \\
\mathrm{m} / \mathrm{s}\end{array}$ & $\begin{array}{l}1.15(1.00-1.23) \\
\mathrm{m} / \mathrm{s}\end{array}$ & $\begin{array}{l}0.78(0.69-0.81) \\
\mathrm{m} / \mathrm{s}\end{array}$ \\
\hline & & & $\mathrm{CV}$ & $24.2(18.4-27.5) \%$ & N/A (N/A-7.6) \% & $23.2(20.3-24.8) \%$ & $15.7(14.1-16.4) \%$ \\
\hline \multirow{2}{*}{$160 \mathrm{mmHg}$} & \multirow[t]{2}{*}{17} & \multirow{2}{*}{$\begin{array}{l}10.77(10.52-11.02) \\
\mathrm{m} / \mathrm{s}\end{array}$} & SD & $\begin{array}{l}1.91(1.73-2.05) \\
\mathrm{m} / \mathrm{s}\end{array}$ & $\begin{array}{l}0.86(0.19-1.08) \\
\mathrm{m} / \mathrm{s}\end{array}$ & $\begin{array}{l}2.09(1.93-2.17) \\
\mathrm{m} / \mathrm{s}\end{array}$ & $\begin{array}{l}1.60(1.40-1.68) \\
\mathrm{m} / \mathrm{s}\end{array}$ \\
\hline & & & $\mathrm{CV}$ & $17.7(15.9-19.3) \%$ & $8.0(1.8-10.0) \%$ & $19.4(17.8-20.3) \%$ & $14.9(13.0-15.6) \%$ \\
\hline \multicolumn{8}{|c|}{ Quasi-static axial stiffness coefficient $\left(c_{\mathrm{ax}}\right)$} \\
\hline \multirow{2}{*}{$10 \mathrm{mmHg}$} & \multirow{2}{*}{19} & \multirow{2}{*}{$1.27(1.24-1.30) \mathrm{g}$} & SD & $0.25(0.22-0.27) \mathrm{g}$ & $0.09(\mathrm{~N} / \mathrm{A}-0.13) \mathrm{g}$ & $0.26(0.24-0.28) \mathrm{g}$ & $0.20(0.17-0.21) \mathrm{g}$ \\
\hline & & & $\mathrm{CV}$ & $19.6(17.0-21.6) \%$ & $6.8(\mathrm{~N} / \mathrm{A}-10.1) \%$ & $20.8(19.0-21.7) \%$ & $15.4(13.4-16.3) \%$ \\
\hline \multirow{2}{*}{$60 \mathrm{mmHg}$} & \multirow{2}{*}{19} & \multirow{2}{*}{$1.30(1.27-1.33) \mathrm{g}$} & SD & $0.26(0.24-0.29) \mathrm{g}$ & $0.07(\mathrm{~N} / \mathrm{A}-0.11) \mathrm{g}$ & $0.27(0.23-0.30) \mathrm{g}$ & $0.20(0.15-0.22) \mathrm{g}$ \\
\hline & & & $\mathrm{CV}$ & $20.3(18.3-21.9) \%$ & $5.1(\mathrm{~N} / \mathrm{A}-8.4) \%$ & $20.9(18.1-22.5) \%$ & $15.2(11.7-16.6) \%$ \\
\hline \multirow{2}{*}{$100 \mathrm{mmHg}$} & \multirow{2}{*}{19} & $265(258-271)$ & SD & $0.38(0.32-0.43) \mathrm{g}$ & $0.35(0.28-0.38) \mathrm{g}$ & $0.52(0.46-0.55) \mathrm{g}$ & $0.44(0.39-0.47) \mathrm{g}$ \\
\hline & & $2.65(2.58-2.71) \mathrm{g}$ & $\mathrm{CV}$ & $14.4(12.1-16.0) \%$ & $13.4(10.8-14.5) \%$ & $19.6(17.5-20.7) \%$ & $16.8(14.8-17.6) \%$ \\
\hline $140 \mathrm{~mm} \mathrm{Ho}$ & 19 & $09-545)$ & SD & $0.46(0.40-0.52) \mathrm{g}$ & $1.13(0.96-1.22) \mathrm{g}$ & $1.23(1.07-1.30) \mathrm{g}$ & $1.18(1.01-1.26) \mathrm{g}$ \\
\hline $140 \mathrm{mmHg}$ & 19 & $5.27(5.09-5.45) \mathrm{g}$ & $\mathrm{CV}$ & $8.8(7.6-9.7) \%$ & $21.5(18.3-22.9) \%$ & $23.3(20.5-24.4) \%$ & $22.4(19.4-23.7) \%$ \\
\hline 200 & 19 & $10.48(10.13-10.83)$ & SD & $0.84(0.77-0.90) \mathrm{g}$ & $2.26(1.93-2.41) \mathrm{g}$ & $2.41(2.11-2.55) \mathrm{g}$ & $2.33(2.02-2.48) \mathrm{g}$ \\
\hline $200 \mathrm{mmHg}$ & 19 & $\mathrm{~g}$ & $\mathrm{CV}$ & $8.1(7.4-8.6) \%$ & $21.5(18.6-22.8) \%$ & $23.0(20.3-24.1) \%$ & $22.3(19.4-23.5) \%$ \\
\hline
\end{tabular}

Table 1. Repeatability statistics. $n_{\text {sample: }}$ number of included samples. Only samples which were measured in duplicate are included in this analysis (hence $2 n_{\text {sample }}$ : number of included measurements). Values between parentheses indicate the $25-75 \%$ confidence interval, obtained using (nonparametric) percentile bootstrapping. Within-subject and between-subject SDs based on analysis described by Rodbard ${ }^{67}$. N/A not applicable; imaginary number arising from a negative estimated between-subject variance caused by sampling error. Composite SDs $(s)$ represent the expected 'between-sample variation' for an unknown sample of $n_{\text {rep }}$ replicates, and were calculated using $s=\sqrt{s_{\mathrm{b}}^{2}+s_{\mathrm{w}}^{2} / n_{\text {rep }}}$, with $s_{\mathrm{b}}$ and $s_{\mathrm{W}}$ the between- and within-subject SD, respectively. This equation can also be used to estimate expected SDs for any $n_{\text {rep }}$, which may be useful for power calculations for future studies. ${ }^{*}$ For quasi-static PWV at $120 \mathrm{mmHg}, \mathrm{s}_{\mathrm{b}}^{2}=-0.11 \mathrm{~m}^{2} / \mathrm{s}^{2}$.

Quasi-static axial extension. The axial stiffness coefficient increased significantly with increasing pressure $\left(\chi^{2}(4)=76.2, p<0.001\right)$. Dunn-Bonferroni post hoc tests revealed the axial stiffness coefficient increased with increasing pressure (1.3 [1.1-1.4], 1.3 [1.2-1.4], 2.6 [2.2-2.8], 5.5 [4.3-6.1], and 10.7 [8.6-11.6] g, respectively) between all groups $(p<0.05)$ but not between 10 and 60, 100 and 140 , and 140 and $200 \mathrm{mmHg}(p=1.00$, $p=0.46$ and $p=0.46$ respectively; Fig. 6 ).

Influence of overnight storage. The maximum time between isolation and mechanical testing of the common carotid artery (CCA) was $24 \mathrm{~h}$ in this study. We did not observe any influence of storage up to $24 \mathrm{~h}$ on arterial mechanics (Tables S1-S3). In particular, we did not find a difference in quasi-static PWV between fresh and non-fresh tested vessels at different axial stretches $\left(0.95 \lambda_{z, i v}, p=0.80 ; 1.00 \lambda_{z, i v}, p=0.65 ; 1.05 \lambda_{z, i v}, p=0.052\right)$, during quasi-static inflation experiments $(80 \mathrm{mmHg}, p=0.65 ; 120 \mathrm{mmHg}, p=1.00 ; 160 \mathrm{mmHg}, p=0.65)$, or dynamic inflation experiments $(80 \mathrm{mmHg}, p=0.28 ; 120 \mathrm{mmHg}, p=0.86 ; 160 \mathrm{mmHg}, p=0.47)$. In addition, axial stiffness coefficients did not differ between fresh and non-fresh tested vessels $(10 \mathrm{mmHg}, p=0.66 ; 60 \mathrm{mmHg}$, $p=1.00 ; 100 \mathrm{mmHg}, p=0.18 ; 140 \mathrm{mmHg}, p=0.66 ; 200 \mathrm{mmHg}, p=0.66)$.

Difference between left and right common carotid arteries. PWV between left and right carotid arteries differed at $\lambda_{z}=\lambda_{z, i v}$ and $120 \mathrm{mmHg}$ for quasi-static inflation experiments (4.4 [4.1-4.9] vs. 5.4 [4.7-5.8] 
A

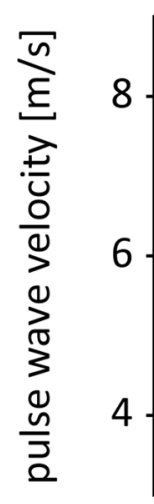

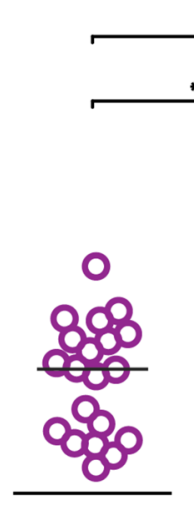

0.95

C

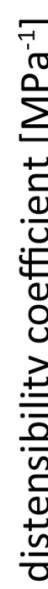

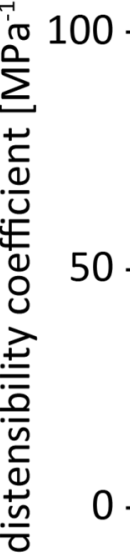

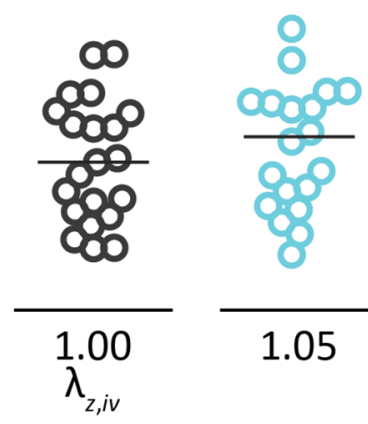

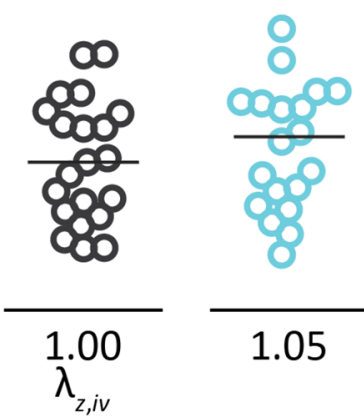

1.05
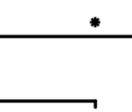

B

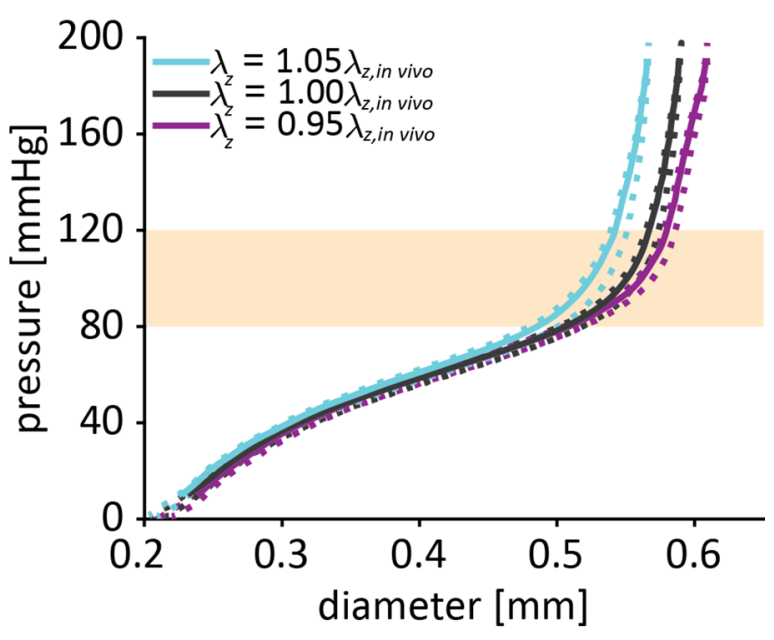

D

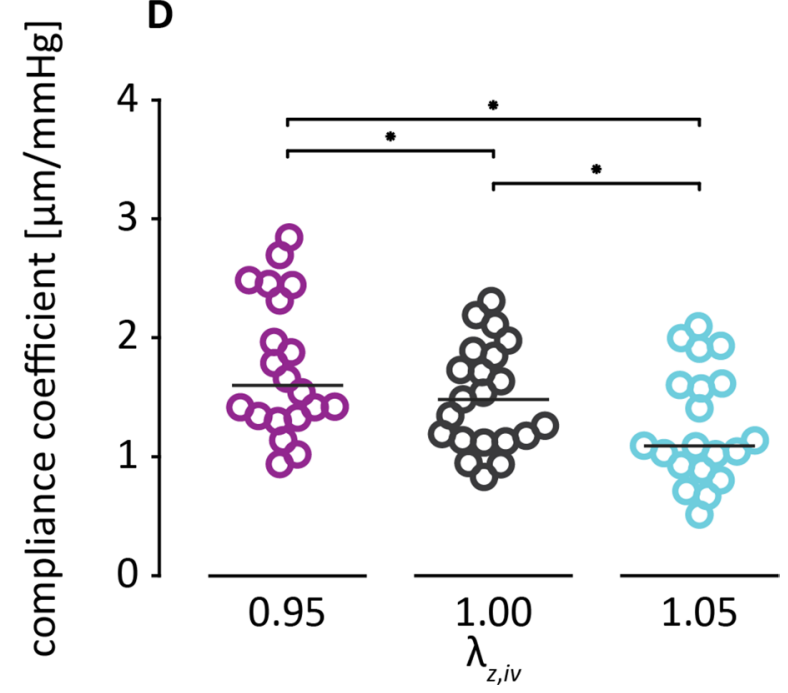

Figure 4. Quasi-static arterial stiffness increased with increasing stretch. (A) pulse wave velocity (PWV) determined from quasi-static diameter relationships for different stretch ratios. PWV was determined at 120/80 mmHg (orange area in (B)). Lines indicate median PWV. (B) Representative example of quasi-static pressure-diameter relationships from one vessel at different stretch ratios. Dashed lines reflect the pressurediameter relation during inflation (ascending line) and deflation (descending line), the straight line reflects the average. (C) Distensibility coefficient (DC) determined from quasi-static pressure-diameter curve at reference pressure 120/80 mmHg. (D) Compliance coefficient (CC) determined from quasi-static pressure-diameter curve at reference pressure $120 / 80 \mathrm{mmHg}{ }^{*} p<0.05, n=20$.

$\mathrm{m} / \mathrm{s}, p=0.04)$. This difference was neither found at $\lambda_{z}=0.95 \lambda_{z, i v}(p=0.58)$, nor at $1.05 \lambda_{z, i v}(p=0.39$; Table S1). There was no significant difference between left and right carotid arteries during quasi-static inflation experiments at $80 \mathrm{mmHg}(p=0.65)$, and $160 \mathrm{mmHg}(p=0.39$; Table S2), nor during dynamic inflation experiments ( $80 \mathrm{mmHg}, p=0.13 ; 120 \mathrm{mmHg} . p=0.86 ; 160 \mathrm{mmHg}, p=0.70$; Table S2). In addition, axial stiffness coefficients did not differ between left and right carotid arteries for all tested pressures $(p=0.39, p=0.97, p=1.00, p=0.97$, and $p=0.48$; Table S3).

\section{Discussion}

In the present study, we present and evaluate a measurement set-up for biomechanical characterisation of murine arteries under pulsatile conditions and at a realistic axial stretch. Measurements showed an acceptable reproducibility, with within-subject CVs of about $20 \%$ (Table 1). We observed a significant difference in PWV between quasi-static and dynamic measurements, as a function of pressure (Fig. 5A). This finding indicates that pulsatile (in comparison to quasi-static) loading indeed affects the biomechanical behaviour of the vessel wall.

Dynamic vs. quasi-static experiments. With our set-up, we aimed to mimic in vivo conditions, using sharply rising pressure pulses reasonably similar to an in vivo blood pressure waveform. At $5 \mathrm{~Hz}(300 \mathrm{bpm})$, 
A
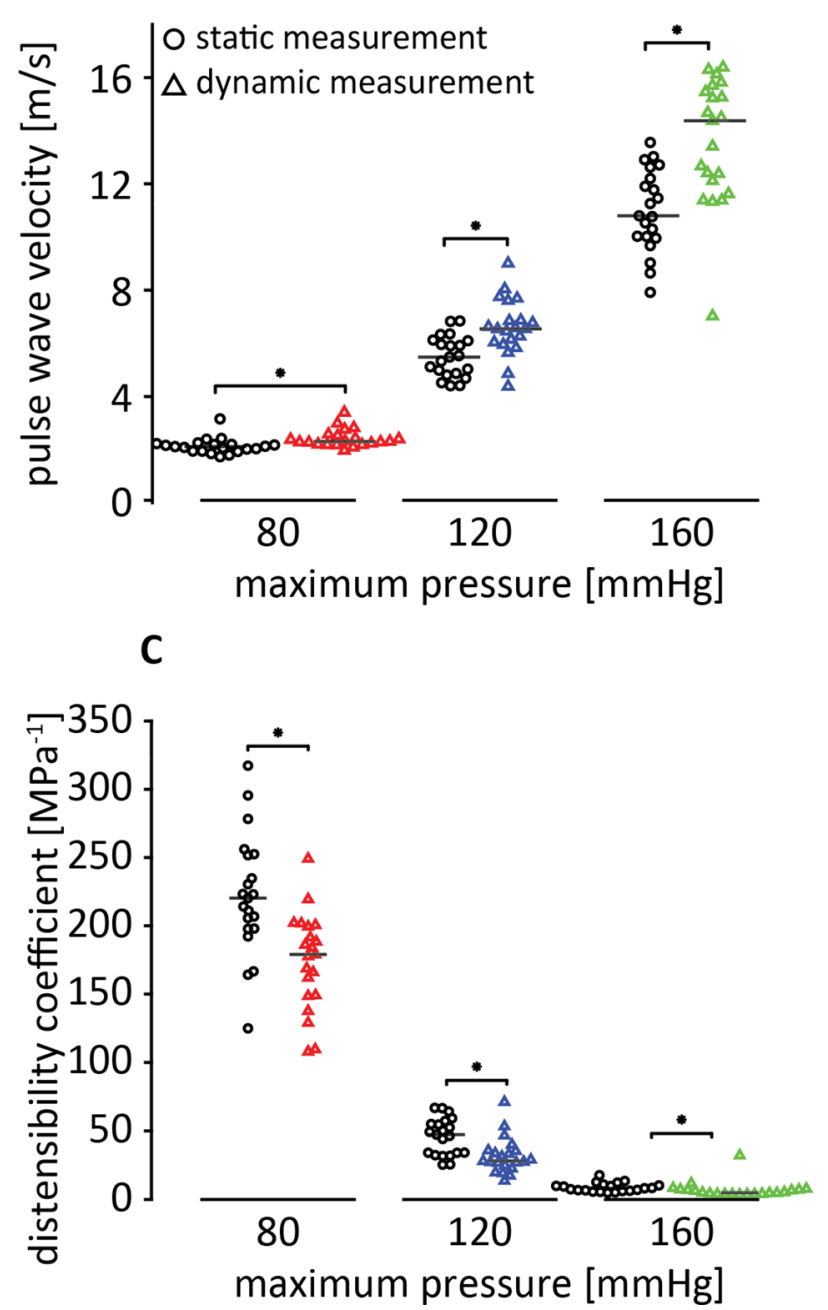

B
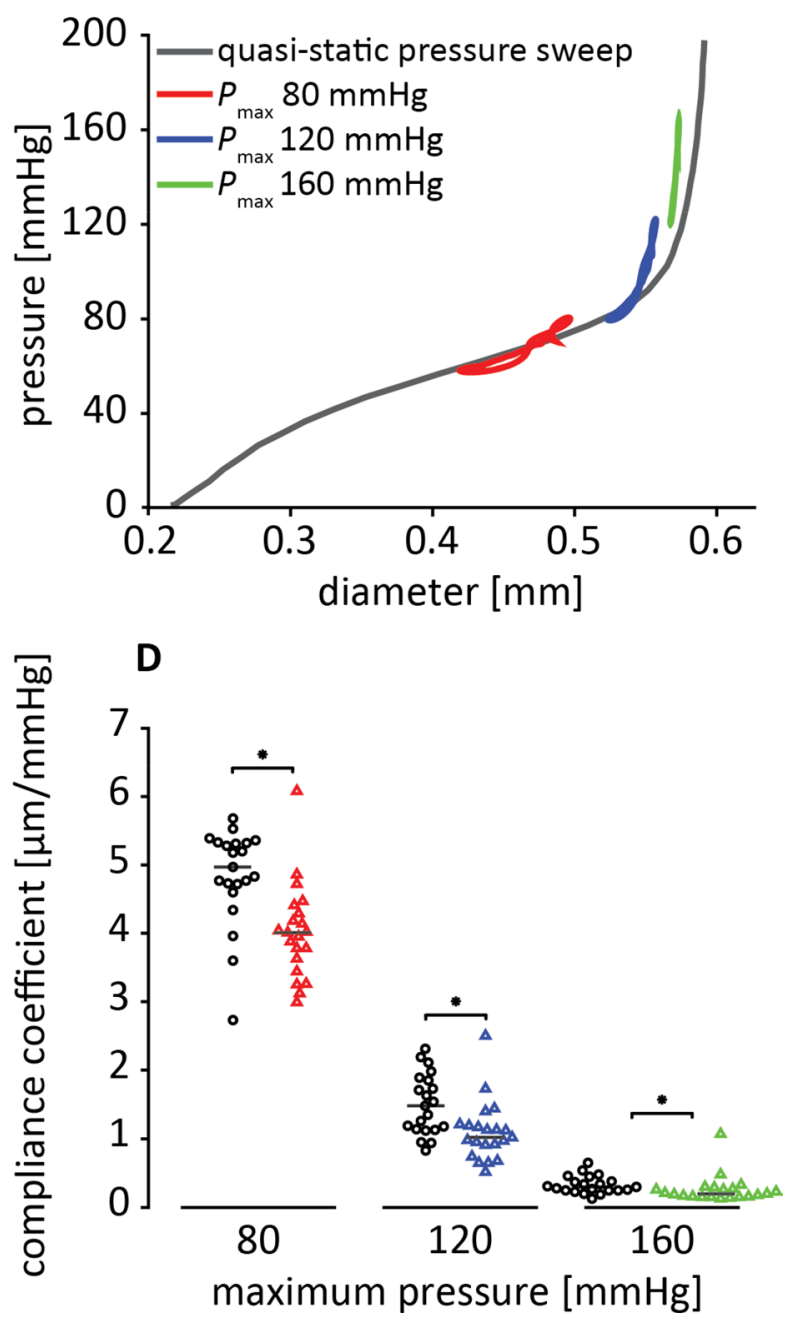

Figure 5. Static experiments underestimate stiffness determined under pulsatile (in vivo) conditions. (A) PWV determined from dynamic pressure-diameter curves compared to the corresponding quasi-static pressurediameter curve. (B) Representative set of quasi-static (grey) and dynamic pressure-diameter curves (the latter obtained for maximum pressures $P_{\max }$ of $80,120,160 \mathrm{mmHg}$ ). (C) Distensibility coefficient (DC) determined from dynamic pressure-diameter curves compared to the corresponding quasi-static pressure-diameter curve. (D) Compliance coefficient (CC) determined from dynamic pressure-diameter curves compared to the corresponding quasi-static pressure-diameter curve. ${ }^{\star} p<0.05, n=21$.

upstroke duration in our system was $\sim 36 \mathrm{~ms}$ (Fig. 3), which better mimics the in vivo rate of circumferential deformation than, e.g., sinusoidal waveforms, where the upstroke duration would be $100 \mathrm{~ms}$ at $5 \mathrm{~Hz}$.

It is important to note our specific pipette and pressure recording configuration (Figs. 1, 2) ${ }^{18,21,26-29}$. This configuration, in conjunction with the zero-phase error processing of pressure and diameter signals, is essential for interpreting observed differences between quasi-static and dynamic behaviour in terms of (viscous) wall behaviour. If the properties of the tested vessel itself would determine the phase and frequency response, the respective contributions of system and vessel could not be disentangled. This would have been the case if we had used $P_{1}$ instead of $P_{2}$ to represent intravascular pressure. Taken together, the design and implementation of our set-up are optimally suited to record dynamic pressure-diameter curves over the large pressure range considered using physiological pulse waveforms (see "Experimental procedures" in "Methods" section).

Our measured dynamic pressure-diameter $(P-d)$ curves did not exhibit significant hysteresis (no substantial net loop area (Fig. 5B)). Dynamic $P$ - $d$ curves at lower mean pressures tended to show more loop-like behaviour, be it in 'figure-8' fashion, with the net loop area remaining small. The negligible loop area we observed is corroborated by $P$ - $d$ curves as measured in vivo in catheterised patients ${ }^{30}$. Although our current data do not indicate visco-elastic behaviour exposed by hysteresis in the dynamic $P$ - $d$ curves, the clear difference in overall steepness of the dynamic curves when compared to the quasi-static $P$ - $d$ curves does signify a strain rate effect, indicative of a viscous component. Others have found comparable differences between dynamic and static elastic behaviour in other experimental animals ${ }^{19,20,27}$. 
A

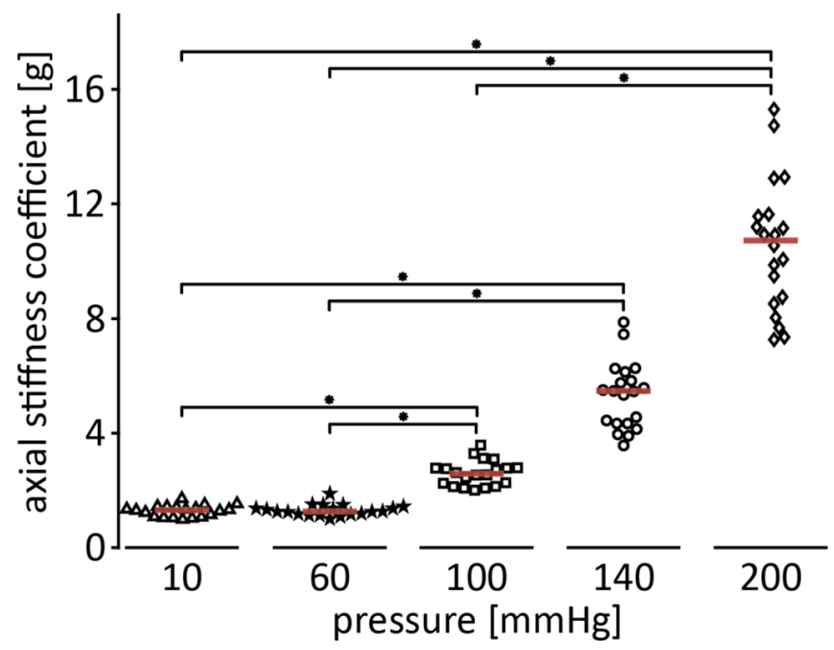

B

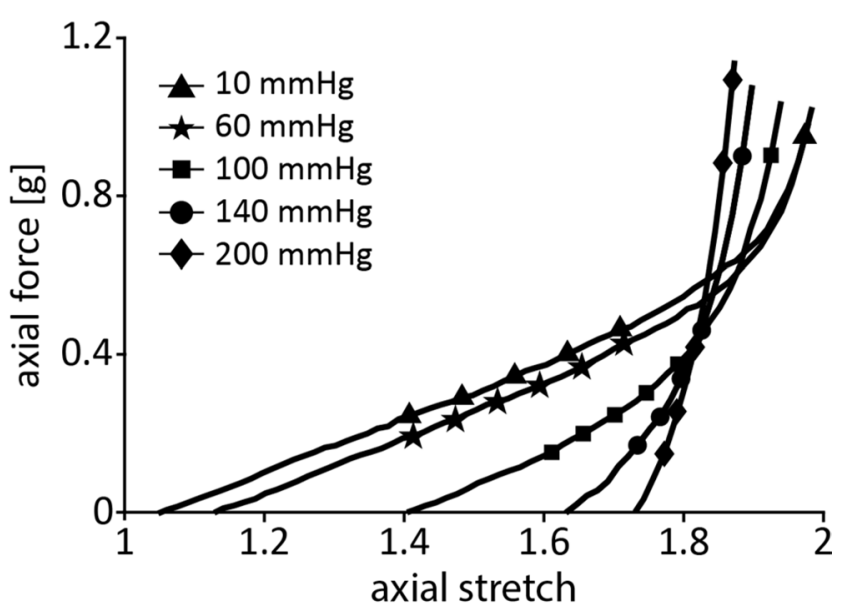

Figure 6. Axial stiffness coefficient increases with increasing pressure. (A) Axial stiffness coefficient was determined as the local derivative at $\lambda_{z}=\lambda_{z, i v}$ (mean crossing points of force-stretch curves (B)). Asterisks indicate statistically significant differences between groups. ${ }^{*} p<0.05, n=20$. (B) Representative quasi-static force-stretch relation under different static pressures.

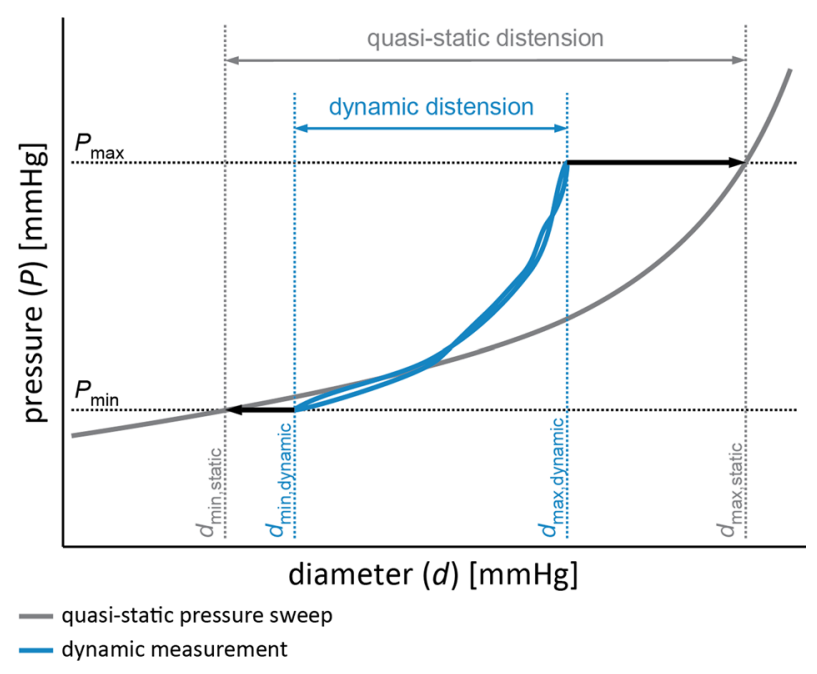

Figure 7. Schematic overview showing how diameters and pressures are obtained for pulse wave velocity calculation for both quasi-static and dynamic experiments. Maximum and minimum pressure ( $P_{\max }$ and $P_{\min }$, respectively) are taken from the dynamic measurement. At these given pressures, the corresponding minimum and maximum diameters $\left(d_{\min }\right.$ and $\left.d_{\max }\right)$ are obtained for the dynamic (blue line) and quasi-static (grey line) diameter recordings.

The deformation response of visco-elastic materials depends both on the amount of force applied (elastic response) and on its rate of change (viscous response) ${ }^{27}$. This rate of change differs between the dynamic and static inflation experiments. This translates into a smaller distension under dynamic than under static conditions, leading to a higher PWV (Eq. (1); Figs. 5A, 7). We conclude that the difference in static and dynamic PWVs is a result, at least partly, of viscous behaviour. The difference in PWV between dynamic and static experiments increases with increasing pressure (Fig. 5A). This could be due to intrinsic phenomena which are not explained by viscous behaviour only. For example, there might be residual vascular smooth muscle cell (VSMC) reactivity, which apparently is more triggered at higher pressure ranges ${ }^{31,32}$. Although we suppressed active contribution of VSMCs to viscous behaviour by adding sodium nitroprusside (SNP) to the buffer, this might have not been sufficient. Inactivation of VSMC by using stronger substances such as ethylenediaminetetraacetic acid (EDTA) could be performed in the future to check the effect of our current choice for SNP ${ }^{33,34}$.

In this study, we calculated and reported PWV based on the Bramwell-Hill equation, DC, and CC as measures of structural vascular stiffness. The use of other, large-strain stiffness metrics could potentially lead to different results and conclusions ${ }^{25}$. In the future, we aim to use our set-up for a full, constitutive characterization of arterial 
tissue $^{11}$, also yielding estimates of material stiffness. Such approach necessitates calculation of stress (force per area) and hence requires a measurement of wall thickness. Wall thickness measurements can easily be obtained by cutting a ring from the artery after the experiment and measuring its thickness or cross-sectional area under a low-magnification microscope ${ }^{11}$. Using such thickness, outer diameter can be easily calculated from inner diameter at any stretch state (using conservation of volume). Because of the availability of pulsatile data, we will be able to extend the current standard approach (modelling the artery hyper elastically using a combination of an isotropic and fibrous component) with descriptors for visco-elasticity (e.g. quasi-linear viscoelasticity). Such studies would allow for quantification of differences in arterial viscoelasticity on a constitutive level in diseases such as diabetes, hypertension, or chronic kidney disease.

Reproducibility. Our measurements showed an acceptable reproducibility, with within-subject CVs around $20 \%$ (Table 1). Sample size calculations for future studies aiming to show a treatment effect of $2 \mathrm{~m} / \mathrm{s}$ result in a required $n=9$ animals per group for dynamic PWV measurements at $120 \mathrm{mmHg}$ (standard deviation $(\mathrm{SD})=1.41 \mathrm{~m} / \mathrm{s}$ ). In case measurements are performed in duplicate (as e.g. in the present study), this reduces to $n=6(\mathrm{SD}=1.10 \mathrm{~m} / \mathrm{s})$.

In this study, the maximum time between isolation and mechanical testing of the CCA was $24 \mathrm{~h}$. Although we did not perform active contraction/dilatation experiments, Gleason et al. reported that a murine carotid artery's response to vasodilators and vasoconstrictors can be maintained for up to four days after isolation when cultured appropriately ${ }^{10}$.

In vivo axial stretch determination. As the axial stretch of an artery influences the distension measured under both quasi-static and dynamic conditions, it is important to determine the in vivo axial stretch as accurately as possible $e^{27,35}$. In the present study, this in vivo axial stretch was estimated as the decoupling stretch between axial force and pressure ${ }^{36,37}$. In the current experimental protocol, at the in vivo axial stretch determination step, the vessel is rapidly (in about $1 \mathrm{~s}$ ) inflated from 0 to $140 \mathrm{mmHg}$ and the difference in axial force between those pressures is used to identify (deviation from) the "experimental" in vivo axial stretch $\left(\lambda_{z, i v}\right)$. During data analysis (retrospectively; in silico), the in vivo axial stretch is recalculated as the mean of crossing points of the quasi-static axial force-stretch curves at $60,100,140$, and $200 \mathrm{mmHg}$. The latter metric $\left(\lambda_{z, i v, \text { calc }}\right.$ ${ }_{11,38}$ ) is estimated using much more data and, hence, more robust (e.g., against noise). $\lambda_{z, i v}$ and $\lambda_{z, i v, \text { calc }}$ differ slightly from each other (Fig. S1). In a future protocol, gradual inflation/deflation while recording axial force could potentially enable more precise determination of the in vivo axial stretch already at the experimental stage.

In the present study, we did not assess how $\lambda_{z, i v} / \lambda_{z, i v \text {,calc }}$ compare to the "actual" in vivo axial stretch as would be obtained from measuring the amount of retraction in situ at the time of excision $\left(\lambda_{z, i s}\right)$. Previous studies observed that for the carotid artery, $\lambda_{z, i v}$ and $\lambda_{z, i s}$ agree well ${ }^{37,38}$. However, this is not the case for all arteries, with e.g. Schulze-Bauer et al. observing $\lambda_{z, \text { is }}$ to be substantially smaller than $\lambda_{z, i v}{ }^{39}$ in the human iliac artery, and Jadidi et al. observing a similar distance in femoropopliteal arteries of young humans ${ }^{40}$. Taken together, when using our set-up to test arteries other than the carotid, care should be taken not to directly assume $\lambda_{z, i v} / \lambda_{z, i v \text {,calc }}$ to be representative of $\lambda_{z, i \text { s }}$, to avoid the risk of tissue damage due to supraphysiological stretching and to avoid misinterpreting the obtained results as representative of the in vivo situation.

Limitations. Under typical awake conditions, murine heart rate is $\sim 600 \mathrm{bpm}$. In the current study, we performed our experiments at a pulse frequency of $5 \mathrm{~Hz}(300 \mathrm{bpm})$, which corresponds approximately to a murine heart rate under general anaesthesia ${ }^{41}$. As the measured biomechanical behaviour of the artery may vary with pulse frequency ${ }^{42}$, we aim to perform future experiments also at other pulse frequencies, e.g. 2.5 and $10 \mathrm{~Hz}$.

The translation of animal experimental research to the human situation remains challenging. Murine and human arteries contain the same structures, but their pulsatile loading rates differ (500-700 bpm in mice compared to 60-100 bpm in humans). Heart rate dependency of PWV has been studied in pacing experiments in both human and rat arteries ${ }^{43-45}$. Relative changes in PWV that were obtained at different heart rates were very similar between the two studies, despite the different species. Tan et al. report an allometric relationship where heart rate is inversely related to body mass ${ }^{44}$. Such relationship could be used to translate findings for a large range of species, including murine experiments, to the human situation.

To minimise the number of animals used in research, we used surplus mice from our animal facility. This has the disadvantage that we did not have control over the animals' background. In addition, we did not receive any detailed information on age or interventions on the live animals prior to euthanasia. Such use of materials is justified because in the present study, we fully focus on paired, i.e. within-animal comparisons, with appropriate power to assess reproducibility and feasibility to accurately study differences between quasi-static and dynamic stiffness.

With the present findings we focus on the dynamic (pulsatile) loading aspects in the circumferential direction. Large arteries experience little to no axial deformation during the cardiac cycle. The vessels might move in longitudinal direction, but vessels mostly have grown and remodelled to have no change in axial force with increasing pressures at in vivo stretch ${ }^{46-50}$. From a biomechanical point of view, it might be interesting to include axial dynamic measurements in an experimental set-up. However, with the present set-up this is not possible.

In our current experiments, by design, there was no intraluminal flow through the vessels (Figs. 1, 2A). We do acknowledge that in vivo, wall shear stress is an important determinant of endothelial-derived factors that regulate vascular tone which influences long-term remodelling ${ }^{51}$. However, our set-up is not built to study long-term structural changes but to characterise biomechanical arterial wall properties under pulsatile pressure loading. Developing a system that, besides a realistic pressure loading, also simultaneously shows a realistic flow loading profile (i.e. at murine pulse rate, with solution of appropriate viscosity) is not trivial ${ }^{20}$ and, 
to our knowledge, has not been performed for murine-size vessels. Giezeman et al. designed an elegant set-up enabling a steady-state flow independent of sinusoidal variations in transmural pressure. However, as detailed and discussed (see "Choice of pressure measurement location to represent intravascular pressure" in "Methods" section, and "Dynamic vs. quasi-static experiments" in "Discussion" section) pipette flow through a pipette will cause errors in the measurement of the transmural pressure waveform, which are of critical importance when assessing visco-elastic vessel properties.

Outlook and relevance. Blood pressure and age are established as two major determinants of arterial stiffness $^{52}$. There remains an ongoing discussion on the effect of heart rate on vascular stiffness ${ }^{42,45,53,54}$. In the present study, we were able to show the feasibility of our set-up for quantifying blood pressure- and loading rate-dependencies of arterial stiffness. The role of heart rate on arterial stiffness measurements can be further elaborated within this set-up.

In future, data generated using our set-up can be used to characterise constitutive models of the artery wall ${ }^{12,55-57}$. Such models can be used to interpret the obtained measurements, yielding insight into the mechanical behaviour and contributions of the individual wall components. Such analyses may contribute to better understanding of factors that contribute to arterial stiffness, which may eventually result in novel treatment opportunities.

The set-up we developed fits directly under a two-photon laser scanning microscope. This would allow imaging and quantification of structural wall components under the same loading conditions as our mechanical testing. Results from such measurements, hence, can potentially be used to directly inform mechanical (constitutive) models at the corresponding loading states, enabling precise studies of the structure-function relationships across the tissue and vessel scales.

Conclusions. We demonstrated the feasibility of our integrated set-up for ex vivo biomechanical characterisation of passive biaxially-loaded murine carotid arteries under pulsatile conditions, with an acceptable reproducibility for practical application. In the present study, we have extended to murine carotid arteries the observation known from studies in other animals that arteries behave stiffer under pulsatile (in vivo) conditions than under quasi-static conditions. This set-up enables further detailed research on how vessel wall components affect arterial biomechanics. This knowledge is important in unravelling (abnormal) arterial stiffening, and associated cardiovascular consequences.

\section{Methods}

Experimental set-up. Our experimental set-up (Fig. 1) is designed to assess biomechanical characteristics of murine carotid arteries and other blood vessels of similar dimensions (i.e. loaded length $\sim \mathrm{mm}$, loaded diameter $\sim 0.7 \mathrm{~mm}$ ), under quasi-static as well as pulsatile conditions. The set-up is a closed-loop system, which enables it to run for a prolonged period of time to cyclically distend the mounted artery while re-using fluid for distension.

Fluid circuit. The bulk of the system's fluid is contained in the vessel bath $(\sim 65 \mathrm{ml})$ and the fluid reservoir $(\sim 15 \mathrm{ml})$. Both are temperature controlled to $37^{\circ} \mathrm{C}$. Reservoir fluid is pressurised using compressed air; air pressure is regulated using a computer-controlled regulator (Bronkhorst IQ + FLOW, Bronkhorst High-tech BV, Ruurlo, The Netherlands; interfaced through universal serial bus (USB)). Reservoir pressure is measured using a pressure sensor $\left(P_{0}\right.$, Fig. 1$)$ and determines the maximum steady-state transmural pressure of the mounted vessel segment; fluid in the vessel bath is exposed to ambient air and hence at atmospheric pressure. An optical level sensor detects when reservoir fluid lever drops below $\sim 60 \%$, starting the return pump to draw fluid from the vessel bath back into the reservoir.

From the reservoir, a short pipe leads to a compliant tube, indenter and a pulse generator (Fig. 1). With each pulse a small volume of fluid flows from the reservoir into a hydraulic resistance-compliance (RC) circuit. The main fluid resistance of this circuit (Fig. 1) is governed by a small multi-turn valve; through this valve fluid flows into the vessel bath. System compliance is distributed and arises from the use of compliant silicone tubing throughout the system (Fig. 1). The resulting pressure waveform is sharply rising and reasonably similar to an in vivo pressure waveform (Fig. 3). The RC circuit is connected to the proximal pipette (p, Fig. 1) via a flowthrough pressure sensor $\left(P_{1}\right.$, Fig. 1). The distal pipette (d, Fig. 1) is connected to another flow-through pressure sensor $\left(P_{2}\right)$, of which the outflow end is closed during experiments. By de-airing and closing the outflow end of $P_{2}$, there is no flow across the distal pipette, rendering the distal pipette to act as an extension of $P_{2}$, causing $P_{2}$ to capture intraluminal pressure with negligible phase and amplitude errors over a large bandwidth (also see "Lumped-parameter modelling of pressure sensor configurations" section below).

Vessel bath and ultrasound. The two custom-drawn glass pipettes (outer diameter $\sim 400 \mu \mathrm{m}$ ) between which the vessel segment is mounted, are submerged in the vessel bath. The proximal pipette (p, Fig. 1) is attached to a slide, allowing motorised axial stretch setting (by stepper motor). The distal pipette (d, Fig. 1) is attached to a load cell, facilitating axial force measurement.

Mounted vessels are imaged longitudinally from the top using a high-frequency ultrasound transducer (MS700, FujiFilm VisualSonics Inc., Toronto, ON, Canada), connected to a VEVO 2100 system. The transducer was fixed in a holder throughout the protocol, ensuring imaging plane consistency between measurements. Acquisition was performed in free running (no triggering) B-mode, which, using our settings (Table S4), resulted in a frame rate of $564 \mathrm{~Hz}$. For each pulsatile recording, 1000 consecutive frames are acquired. 


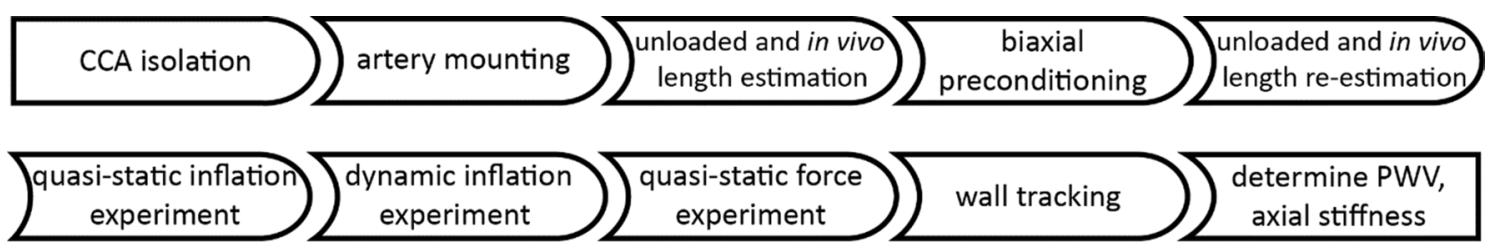

Figure 8. Timeline of experimental protocol. $C C A$ common carotid artery, $P W V$ pulse wave velocity.

Control of indenter and motorised slide. An Arduino UNO microcontroller (Arduino, Somerville, MA, USA; interfaced through USB) is used to control the pulse generator and stepper motor (axial stretch). In addition, synchronously with each pressure pulse, the microcontroller generates an electrical synchronisation pulse (see "Signal acquisition and control" section).

Signal acquisition and control. The following analogue signals are acquired by a USB-6001 A/D-D/A converter (National Instruments Corporation, Austin, TX, USA; interfaced through USB) at a sampling rate of $2500 \mathrm{~Hz}$ : pressures $P_{0}, P_{1}$, and $P_{2}$; temperatures of reservoir and vessel bath; axial force; and the synchronisation signal. The level sensor is connected to a digital input of the A/D-D/A converter; the heating circuits of the reservoir and vessel bath, and the return pump are driven from three digital outputs.

Signal acquisition and control are performed through a custom LabVIEW interface (LabVIEW 2013, National Instruments Corporation, Austin, TX, USA). Importantly, the synchronisation signal is also fed to the ECG input of the VEVO 2100 ultrasound system, facilitating synchronisation of pressure signals and ultrasound acquisitions.

Lumped-parameter modelling of pressure sensor configurations. We performed lumped-parameter modelling to substantiate the relevance of our pipette and pressure recording configuration, in conjunction with zero-phase error processing of pressure and diameter signals. The simulation code is included in Supplemental Digital Content 1. Parameterisation was as follows: Proximal pipette flow resistance was determined from timed volume collection at a known pressure gradient and was about $1 \times 10^{11} \mathrm{~Pa} /\left(\mathrm{m}^{3} / \mathrm{s}\right)$. From preliminary experiments we estimated the compliance of a vessel under test to be about $5 \times 10^{-14} \mathrm{~m}^{3} / \mathrm{Pa}$ at 'normal' physiological pressure conditions, but considerably greater at low pressure conditions: about threefold. The resultant $R C$ time under physiological pressure conditions hence was $5 \mathrm{~ms}$ (simulation code in Supplemental Digital Content 1: in the model this is parameterised by an arbitrary $R_{\text {pipette_prox }}=R_{\text {pipette_dist }}$ of 1 and a $C_{\text {vessel }}$ of 0.005). Using $f_{\mathrm{c}}=1 /(2 \pi R C)$ yields a corner frequency of $32 \mathrm{~Hz}$.

Experimental procedures. An overview of the experimental procedure can be found in Fig. 8 .

Animals. We obtained left and right CCAs from 11 male surplus mice, received from the animal facility of Maastricht University. Animals were euthanised with an overdose of carbon dioxide, after which both CCAs were isolated. One CCA was directly tested, while the other CCA was stored in Hanks' Balanced Salt Solution (HBSS; Thermo Fisher Scientific, Paisley, UK) at $3{ }^{\circ} \mathrm{C}$ for testing the next day (maximum storage time was $24 \mathrm{~h}$ ). We started with the left and right CCA in alternation. All experiments were performed in duplicate, requiring between 3 and $4 \mathrm{~h}$ per vessel. All methods were carried out in accordance with relevant guidelines and regulations. The use of surplus animals, after euthanasia, has been approved by the Maastricht University Animal Ethical Committee.

Preparations. To prime the system, the vessel bath was filled with HBSS, with the return pump used to pump this fluid into the reservoir. To achieve maximal vasodilatation, $10 \mu \mathrm{M}$ of SNP (Sigma-Aldrich, St. Louis, MO, USA) was added to the vessel bath. After filling the entire system, air bubbles were removed and the dead-end tubing of $P_{2}$ was closed off to ensure null compliance distal to the distal pipette (Fig. 1).

Preconditioning. After priming the circuit and mounting the vessel, the vessel was set to its unloaded length. This was done by increasing the length of the mounted vessel in 100- $\mathrm{m}$ steps until the segment appeared straight (i.e. no visually obvious buckling, but not under tension), at negligible transmural pressure. Axial force was then calibrated to read zero. Subsequently, an estimate of the axial in vivo stretch was determined during preconditioning $\left(\lambda_{z, i v, \text { prec }}\right)$ as the stretch at which axial force remains constant during pressurisation from 0 to $140 \mathrm{mmHg}^{36,37,58}$. To eliminate excessive hysteresis and restore wall component rearrangements after excision, the artery was pre-conditioned circumferentially (4 inflation-deflation cycles from 0 to $200 \mathrm{mmHg}$; cycle time $\sim 10 \mathrm{~s}$ ) and axially ( 4 stretch-relaxation cycles between force zero and $F_{\text {max,prec }}$; step size $50 \mu \mathrm{m}$; $P_{2}=100 \mathrm{mmHg}$ ) before the start of the experiment ${ }^{18,27,37,59,60} . F_{\text {max,prec }}$ was determined as the measured force at $\lambda_{z}=1.05 \lambda_{z, i v \text {,prec }}$ and $P_{2}=200 \mathrm{mmHg}$. After this two-step pre-conditioning protocol, the resting length and in vivo axial stretch $\left(\lambda_{z, i v}\right)$ were re-estimated using the method described above. This (re-estimated) $\lambda_{z, i v}$ was used as a starting point for further experiments (Fig. 8).

Quasi-static inflation experiments. Initially, the artery was tested quasi-statically at $\lambda_{z}=\lambda_{z, \text { iv }}$ by measuring inner diameter at small pressure increments from 0 to $200 \mathrm{mmHg}$ and back to $0 \mathrm{mmHg}$, in steps of $5 \mathrm{mmHg}$. Time between steps was $\sim 5 \mathrm{~s}$. We repeated the experiment with $\lambda_{z}$ decreased by $5 \%\left(\lambda_{z}=0.95 \lambda_{z, i v}\right)$ and 
increased by $5 \%\left(\lambda_{z}=1.05 \lambda_{z, i v}\right)$. During the experiment at $1.05 \lambda_{z, i v}$, the axial force at $P_{2}=200 \mathrm{mmHg}$ was recorded as $F_{\max }$ (similar to the preconditioning protocol; see above).

Dynamic inflation experiments. To start the second experiment, $\lambda_{z}$ was set back to $\lambda_{z, i v}$. Subsequently, the pulse generator was activated at $5 \mathrm{~Hz}(300 \mathrm{bpm})$. Reservoir pressure and fluid resistance were tuned such that the maximum and minimum pressure of each pulse cycle as recorded by $P_{2}$ were approximately 120 and $80 \mathrm{mmHg}$, respectively. Vessel distension was simultaneously recorded through ultrasound. After this measurement, reservoir pressure was tuned to result in maximum pressures of $80 \mathrm{mmHg}$ and $160 \mathrm{mmHg}$ respectively, to record pulsatile artery distension at typical hypotensive and hypertensive peak pressures.

Quasi-static stretch experiments. Finally, axial stiffness was assessed quasi-statically, by measuring axial force at small $\lambda_{z}$ steps $\left(\Delta \lambda_{z}\right)$. Axial stretch was increased from resting stretch (i.e. $\lambda_{z}$ for which $F=0$ ) up to $F_{\max }$ in steps of $\Delta \lambda_{z}=0.015$ and then decreased back to resting stretch with the same step size. This experiment was performed at static transmural pressures of 10, 60, 100, 140, and $200 \mathrm{mmHg}$. Diameter assessment was not possible during these measurements as the ultrasound probe did not physically fit between the micropipettes at the resting length. Hence, we did not obtain diameter information in this part of the protocol.

Data processing and calculations. B-mode ultrasound images were processed using B-mode edge tracking as described by Steinbuch et al. ${ }^{61}$. Dynamic pressure and diameter signals were resampled off-line at $1000 \mathrm{~Hz}$. Data were filtered using a 51-point Savitzky-Golay filter of order $8(N=8, M=25)$, having a $-3 \mathrm{~dB}$ cutoff frequency of $\sim 60 \mathrm{~Hz}^{62}$. At a pulse frequency of $5 \mathrm{~Hz}$, this leads to inclusion of the first 12 harmonics of the signals, yielding an accurate representation of the actual signals ${ }^{63,64}$.

We chose to quantify structural vascular stiffness by means of the pulse wave velocity (PWV) because this allows comparison with in vivo vascular stiffness measures. PWV was derived from the Bramwell-Hill relationship:

$$
\mathrm{PWV}=\sqrt{\frac{1}{2 \rho} \times \frac{P_{\max }-P_{\min }}{d_{\max }-d_{\min }} \times d_{\min }},
$$

with $\rho$ the blood mass density, $P_{\min }$ and $P_{\max }$ the minimum and maximum pressures, and $d_{\min }$ and $d_{\max }$ the minimum and maximum inner diameters (Fig. 7). Vessel inner diameter was determined with an operatorindependent MATLAB script (MATLAB R2019a, MathWorks, Natick, MA, USA), to trace the exact location of the complete vessel wall for every timeframe ${ }^{65}$.

Because distensibility and compliance are measures often reported in ex vivo studies, we calculated those for the quasi-static - as well as the dynamic inflation experiments. However, repeatability statistics were only performed with our main outcome variable, PWV.

The distensibility coefficient was calculated as:

$$
\mathrm{DC}=2 \times \frac{d_{\max }-d_{\min }}{d_{\min } \times\left(P_{\max }-P_{\min }\right)} .
$$

Compliance coefficient was calculated from:

$$
\mathrm{CC}=\frac{d_{\max }-d_{\min }}{P_{\max }-P_{\min }} .
$$

To quantify arterial structural stiffness in the axial direction, an in vivo axial stiffness coefficient $c_{\mathrm{ax}}$ (in grams) was calculated as the local derivative of the force-stretch curve for every static pressure:

$$
c_{\mathrm{ax}}=\left.\frac{\mathrm{d} F}{\mathrm{~d} \lambda_{z}}\right|_{\lambda_{z}=\lambda_{z, i v, \text { calc }}} .
$$

The in vivo axial stretch value used in this equation $\left(\lambda_{z, i v, \text { calc }}\right)$ was determined as the mean of crossing points of the quasi-static force-stretch curves at $60,100,140$, and $200 \mathrm{mmHg}$ (Fig. $6^{11}$ ). The force-stretch curve at $10 \mathrm{mmHg}$ morphologically differs substantially from the other curves, and was therefore not included.

Data was processed with a custom MATLAB code.

Statistical analysis. Set-up repeatability was assessed by quantifying within-subject and between-subject SDs and CVs. Within-subject SD was estimated by (1) calculating the sample SD for each set of (two) replicate measurements, (2) averaging the square of these SDs, and (3) taking the square root of the result ${ }^{66,67}$. Subsequently, between-subject SD was estimated in two steps. First, the SD of the subject means was calculated $\left(s_{\bar{x}}\right)$. Note that the value of $s_{\bar{x}}$ is a measure of an inherent "component" of variation between subjects, and in addition, a "component" owing to the measurement error within each subject ${ }^{67}$. Hence, second, between-subject SD $\left(s_{\mathrm{b}}\right)$ was calculated as

$$
s_{\mathrm{b}}=\sqrt{s_{\bar{x}}^{2}-\frac{s_{\mathrm{w}}^{2}}{2}}
$$


Sample size calculations were performed using the MATLAB Statistics and Machine Learning Toolbox at power $1-\beta=0.80$ and $\alpha=0.05$. Data between duplicate measurements of the same vessel were compared with a Wilcoxon signed-rank test for paired measures. To explore the potential influence of vessel freshness and left/right CCA on the outcomes, data from fresh/non-fresh and left/right CCA vessels were compared using a Mann-Whitney U test. All results are expressed as median with corresponding 25th and 75th percentiles and $n$ denoting the number of vessels per group. $p<0.05$ was considered statistically significant. Statistical analyses were performed using IBM SPSS Statistics for Windows (version 24.0; IBM Corp, Armonk, NY).

Received: 25 August 2020; Accepted: 7 December 2020

Published online: 29 January 2021

\section{References}

1. Boutouyrie, P. et al. Aortic stiffness is an independent predictor of primary coronary events in hypertensive patients. Hypertension 39, 10-15. https://doi.org/10.1161/hy0102.099031 (2002).

2. Laurent, S. et al. Aortic stiffness is an independent predictor of all-cause and cardiovascular mortality in hypertensive patients. Hypertension 37, 1236-1241. https://doi.org/10.1161/01.HYP.37.5.1236 (2001).

3. Laurent, S. et al. Aortic stiffness is an independent predictor of fatal stroke in essential hypertension. Stroke 34, 1203-1206. https ://doi.org/10.1161/01.STR.0000065428.03209.64 (2003).

4. Meaume, S., Benetos, A., Henry, O. F., Rudnichi, A. \& Safar, M. E. Aortic pulse wave velocity predicts cardiovascular mortality in subjects $>70$ years of age. Arterioscler. Thromb. Vasc. Biol. 21, 2046-2050. https://doi.org/10.1161/hq1201.100226 (2001).

5. Bonarjee, V. V. S. Arterial stiffness: A prognostic marker in coronary heart disease. Available methods and clinical application. Front. Cardiovasc. Med. 5, 64-64. https://doi.org/10.3389/fcvm.2018.00064 (2018).

6. Ecobici, M. \& Stoicescu, C. Arterial stiffness and hypertension-Which comes first?. Maedica (Bucur) 12, 184-190 (2017).

7. Leloup, A. J. A. et al. A novel set-up for the ex vivo analysis of mechanical properties of mouse aortic segments stretched at physiological pressure and frequency. J. Physiol. 594, 6105-6115. https://doi.org/10.1113/JP272623 (2016).

8. Mitchell, G. F. Arterial stiffness and hypertension: Chicken or egg?. Hypertension 64, 210-214. https://doi.org/10.1161/HYPER TENSIONAHA.114.03449 (2014).

9. Safar, M. E. et al. Interaction between hypertension and arterial stiffness. Hypertension 72, 796-805. https://doi.org/10.1161/ HYPERTENSIONAHA.118.11212 (2018).

10. Gleason, R., Gray, P. S., Wilson, E. \& Humphrey, D. J. A multiaxial computer-controlled organ culture and biomechanical device for mouse carotid arteries. J. Biomech. Eng. 126, 787-795. https://doi.org/10.1115/1.1824130 (2005).

11. Ferruzzi, J., Bersi, M. R. \& Humphrey, J. D. Biomechanical phenotyping of central arteries in health and disease: Advantages of and methods for murine models. Ann. Biomed. Eng. 41, 1311-1330. https://doi.org/10.1007/s10439-013-0799-1 (2013).

12. Holzapfel, G. A. \& Ogden, R. W. Constitutive modelling of arteries. Proc. Math. Phys. Eng. Sci. 466, 1551-1597. https://doi. org/10.1098/rspa.2010.0058 (2010).

13. Lacolley, P., Regnault, V., Segers, P. \& Laurent, S. Vascular smooth muscle cells and arterial stiffening: Relevance in development, aging, and disease. Physiol. Rev. 97, 1555-1617. https://doi.org/10.1152/physrev.00003.2017 (2017).

14. Humphrey, J. D., Milewicz, D. M., Tellides, G. \& Schwartz, M. A. Cell biology. Dysfunctional mechanosensing in aneurysms. Science 344, 477-479. https://doi.org/10.1126/science.1253026 (2014).

15. Cox, R. H. Three-dimensional mechanics of arterial segments in vitro: Methods. J. Appl. Physiol. 36, 381-384. https://doi. org/10.1152/jappl.1974.36.3.381 (1974).

16. Cox, R. H. Arterial wall mechanics and composition and the effects of smooth muscle activation. Am. J. Physiol. 229, 807-812. https://doi.org/10.1152/ajplegacy.1975.229.3.807 (1975).

17. Holzapfel, G. A., Gasser, T. C. \& Stadler, M. A structural model for the viscoelastic behavior of arterial walls: Continuum formulation and finite element analysis. Eur. J. Mech. A Solids 21, 441-463. https://doi.org/10.1016/S0997-7538(01)01206-2 (2002).

18. Learoyd, B. M. \& Taylor, M. G. Alterations with age in the viscoelastic properties of human arterial walls. Circ. Res. 18, 278-292. https://doi.org/10.1161/01.RES.18.3.278 (1966).

19. Lichtenstein, O., Safar, M. E., Mathieu, E., Poitevin, P. \& Levy, B. I. Static and dynamic mechanical properties of the carotid artery from normotensive and hypertensive rats. Hypertension 32, 346-350. https://doi.org/10.1161/01.HYP.32.2.346 (1998).

20. Giezeman, M. J., VanBavel, E., Grimbergen, C. A. \& Spaan, J. A. Compliance of isolated porcine coronary small arteries and coronary pressure-flow relations. Am. J. Physiol. Heart Circ. Physiol. 267, H1190-H1198. https://doi.org/10.1152/ajphe art.1994.267.3.H1190 (1994).

21. Boutouyrie, P. et al. In vivo/in vitro comparison of rat abdominal aorta wall viscosity. Arter. Thromb. Vasc. Biol. 17, 1346-1355. https://doi.org/10.1161/01.ATV.17.7.1346 (1997).

22. Boekhoven, R. W. et al. Inflation and bi-axial tensile testing of healthy porcine carotid arteries. Ultrasound Med. Biol. 42, 574-585. https://doi.org/10.1016/j.ultrasmedbio.2015.09.019 (2016).

23. Hoeks, A. P. G., Willigers, J. M. \& Reneman, R. S. Effects of assessment and processing techniques on the shape of arterial pressuredistension loops. J. Vasc. Res. 37, 494-500. https://doi.org/10.1159/000054082 (2000).

24. Caulk, A. W., Humphrey, J. D. \& Murtada, S.-I. Fundamental roles of axial stretch in isometric and isobaric evaluations of vascular contractility. J. Biomech. Eng. 141, 031008. https://doi.org/10.1115/1.4042171 (2019).

25. Spronck, B. \& Humphrey, J. D. Arterial stiffness: Different metrics, different meanings. J. Biomech. Eng. 141, 091004. https://doi. org/10.1115/1.4043486 (2019).

26. Bauer, R. D. \& Pasch, T. The quasistatic and dynamic circumferential elastic modulus of the rat tail artery studied at various wall stresses and tones of the vascular smooth muscle. Pflügers Arch. 330, 335-346. https://doi.org/10.1007/BF00588585 (1971).

27. Bergel, D. H. The dynamic elastic properties of the arterial wall. J. Physiol. 156, 458-469 (1961).

28. Busse, R., Bauer, R. D., Sattler, T. \& Schabert, A. Dependence of elastic and viscous properties of elastic arteries on circumferential wall stress at two different smooth muscle tones. Pflügers Arch. 390, 113-119. https://doi.org/10.1007/BF00590192 (1981).

29. Greenwald, S. E., Newman, D. L. \& Denyer, H. T. Effect of smooth muscle activity on the static and dynamic elastic properties of the rabbit carotid artery. Cardiovasc. Res. 16, 86-94. https://doi.org/10.1093/cvr/16.2.86 (1982).

30. Hermeling, E. et al. Noninvasive assessment of arterial stiffness should discriminate between systolic and diastolic pressure ranges. Hypertension 55, 124-130. https://doi.org/10.1161/HYPERTENSIONAHA.109.143867 (2010).

31. Leloup, A., De Moudt, S., Van Hove, C. \& Fransen, P. Cyclic stretch alters vascular reactivity of mouse aortic segments. Front. Physiol. 8, 858-858. https://doi.org/10.3389/fphys.2017.00858 (2017).

32. Leloup, A. J. A. et al. Vascular smooth muscle cell contraction and relaxation in the isolated aorta: A critical regulator of large artery compliance. Physiol. Rep. 7, e13934. https://doi.org/10.14814/phy2.13934 (2019).

33. Dupuis, F., Atkinson, J., Limiñana, P. \& Chillon, J.-M. Captopril improves cerebrovascular structure and function in old hypertensive rats. Br. J. Pharmacol. 144, 349-356. https://doi.org/10.1038/sj.bjp.0706001 (2005). 
34. Foulquier, S., Lartaud, I. \& Dupuis, F. Impact of short-term treatment with telmisartan on cerebral arterial remodeling in shr. PLoS ONE 9, e110766. https://doi.org/10.1371/journal.pone.0110766 (2014).

35. Holtackers, R. J. et al. Head orientation should be considered in ultrasound studies on carotid artery distensibility. J. Hypertens. 34, 1551-1555. https://doi.org/10.1097/hjh.0000000000000985 (2016).

36. Brossollet, L. J. \& Vito, R. P. An alternate formulation of blood vessel mechanics and the meaning of the in vivo property. J. Biomech. 28, 679-687. https://doi.org/10.1016/0021-9290(94)00119-O (1995).

37. Weizsäcker, H. W., Lambert, H. \& Pascale, K. Analysis of the passive mechanical properties of rat carotid arteries. J. Biomech. 16, 703-715. https://doi.org/10.1016/0021-9290(83)90080-5 (1983).

38. Van Loon, P. Length-force and volume-pressure relationships of arteries. Biorheology 14, 181-201 (1977).

39. Schulze-Bauer, C. A., Mörth, C. \& Holzapfel, G. A. Passive biaxial mechanical response of aged human iliac arteries. J. Biomech. Eng. 125, 395-406. https://doi.org/10.1115/1.1574331 (2003).

40. Jadidi, M., Desyatova, A., MacTaggart, J. \& Kamenskiy, A. Mechanical stresses associated with flattening of human femoropopliteal artery specimens during planar biaxial testing and their effects on the calculated physiologic stress-stretch state. Biomech. Model. Mechanobiol. 18, 1591-1605. https://doi.org/10.1007/s10237-019-01162-0 (2019).

41. Leloup, A. J. A. et al. Applanation tonometry in mice. Hypertension 64, 195-200. https://doi.org/10.1161/HYPERTENSIONAHA $.114 .03312(2014)$.

42. Spronck, B. et al. Heart rate and blood pressure dependence of aortic distensibility in rats: Comparison of measured and calculated pulse wave velocity. J. Hypertens. https://doi.org/10.1097/hih.0000000000002608 (2020).

43. Lantelme, P., Mestre, C., Lievre, M., Gressard, A. \& Milon, H. Heart rate. Hypertension 39, 1083-1087. https://doi.org/10.1161/01. HYP.0000019132.41066.95 (2002).

44. Tan, I., Butlin, M., Liu, Y. Y., Ng, K. \& Avolio, A. P. Heart rate dependence of aortic pulse wave velocity at different arterial pressures in rats. Hypertension 60, 528-533. https://doi.org/10.1161/HYPERTENSIONAHA.112.194225 (2012).

45. Tan, I., Butlin, M., Spronck, B., Xiao, H. \& Avolio, A. Effect of heart rate on arterial stiffness as assessed by pulse wave velocity. Curr. Hypertens. Rev. https://doi.org/10.2174/1573402113666170724100418 (2017).

46. Cardamone, L., Valentín, A., Eberth, J. F. \& Humphrey, J. D. Origin of axial prestretch and residual stress in arteries. Biomech. Model. Mechanobiol. 8, 431-446. https://doi.org/10.1007/s10237-008-0146-x (2009).

47. Gleason, R. \& Humphrey, J. Effects of a sustained extension on arterial growth and remodeling: A theoretical study. J. Biomech. 38, 1255-1261. https://doi.org/10.1016/j.jbiomech.2004.06.017 (2005).

48. Humphrey, J. D., Eberth, J. F., Dye, W. W. \& Gleason, R. L. Fundamental role of axial stress in compensatory adaptations by arteries. J. Biomech. 42, 1-8. https://doi.org/10.1016/j.jbiomech.2008.11.011 (2009).

49. Patel, D. J., Fry, D. L. \& Janicki, J. S. Longitudinal tethering of arteries in dogs. Circ. Res. 19, 1011-1021. https://doi.org/10.1161/01. RES.19.6.1011 (1966).

50. Tickner, E. G. \& Sacks, A. H. A theory for the static elastic behavior of blood vessels. Biorheology 4, 151-168. https://doi. org/10.3233/BIR-1967-4402 (1967)

51. Jaminon, A., Reesink, K., Kroon, A. \& Schurgers, L. The role of vascular smooth muscle cells in arterial remodeling: Focus on calcification-related processes. Int. J. Mol. Sci. 20, 5694. https://doi.org/10.3390/ijms20225694 (2019).

52. Spronck, B. et al. Pressure-dependence of arterial stiffness: Potential clinical implications. J. Hypertens. 33, 330-338. https://doi. org/10.1097/hjh.0000000000000407 (2015).

53. Xiao, H., Tan, I., Butlin, M., Li, D. \& Avolio, A. P. Arterial viscoelasticity: Role in the dependency of pulse wave velocity on heart rate in conduit arteries. Am. J. Physiol. Heart. Circ. Physiol. 312, H1185-H1194. https://doi.org/10.1152/ajpheart.00849.2016 (2017).

54. Xiao, H., Tan, I., Butlin, M., Li, D. \& Avolio, A. Mechanism underlying heart rate dependency of wave reflection in the aorta: A numerical simulation. Am. J. Physiol. Heart. Circ. Physiol. 314, H443. https://doi.org/10.1152/ajpheart.00559.2017 (2017).

55. Humphrey, J. D. Cardiovascular Solid Mechanics: Cells, Tissues, and Organs (Springer, Berlin, 2011).

56. Spronck, B. et al. A constitutive modeling interpretation of the relationship among carotid artery stiffness, blood pressure, and age in hypertensive subjects. Am. J. Physiol. Heart Circ. Physiol. 308, H568-H582. https://doi.org/10.1152/ajpheart.00290.2014 (2015).

57. Reesink, K. D. \& Spronck, B. Constitutive interpretation of arterial stiffness in clinical studies: A methodological review. Am. J. Physiol. Heart. Circ. Physiol. 316, H693-H709. https://doi.org/10.1152/ajpheart.00388.2018 (2019).

58. Avril, S., Badel, P., Gabr, M., Sutton, M. A. \& Lessner, S. M. Biomechanics of porcine renal arteries and role of axial stretch. J. Biomech. Eng. 135, 81007-81007. https://doi.org/10.1115/1.4024685 (2013).

59. Remington, J. W. Hysteresis loop behavior of the aorta and other extensible tissues. Am. J. Physiol. 180, 83-95. https://doi. org/10.1152/ajplegacy.1954.180.1.83 (1955).

60. Humphrey, J. \& Epstein, M. Cardiovascular solid mechanics: Cells, tissues, and organs. AMR 55, B103-B104. https://doi. org/10.1115/1.1497492 (2002).

61. Steinbuch, J. et al. Standard b-mode ultrasound measures local carotid artery characteristics as reliably as radiofrequency phase tracking in symptomatic carotid artery patients. Ultrasound Med. Biol. 42, 586-595. https://doi.org/10.1016/j.ultrasmedb io.2015.07.030 (2016).

62. Schafer, R. W. What is a savitzky-golay filter? [lecture notes]. IEEE Signal Process. Mag. 28, 111-117. https://doi.org/10.1109/ MSP.2011.941097 (2011).

63. Moxham, I. M. Physics of invasive blood pressure monitoring. S. Afr. J Anaesth. Analg. 9, 33-38. https://doi.org/10.1080/22201 173.2003.10872990 (2014).

64. Vlachopoulos, C., O'Rourke, M. \& Nichols, W. W. Mcdonald's Blood Flow in Arteries, Sixth Edition: Theoretical, Experimental and Clinical Principles (CRC Press, Cambridge, 2011).

65. Hoeks, A. P. G., Brands, P. J., Willigers, J. M. \& Reneman, R. S. Non-invasive measurement of mechanical properties of arteries in health and disease. Proc. Inst. Mech. Eng. H 213, 195-202. https://doi.org/10.1243/0954411991534924 (1999).

66. Bland, J. M. \& Altman, D. G. Measurement error. BMJ (Clin. Res.) 312, 1654-1654. https://doi.org/10.1136/bmj.312.7047.1654 (1996).

67. Rodbard, D. Statistical quality control and routine data processing for radioimmunoassays and immunoradiometric assays. Clin. Chem. 20, 1255 (1974).

\section{Acknowledgements}

We would like to acknowledge the support from the Maastricht University central animal facility.

\section{Author contributions}

Experiments were performed at the Department of Biomedical Engineering and the Muroidean Facility at Maastricht University, Maastricht, the Netherlands. M.M.B., K.D.R., P.J.M.S. and B.S. conceived and designed the experiments. M.M.B., N.B., J.H. and R.T.A.M. performed experiments. M.M.B. and B.S. analysed the data. M.M.B., K.D.R., T.D. and B.S. interpreted the results of experiments. M.M.B. and B.S. prepared figures. M.M.B. and K.D.R. drafted the work. M.M.B., K.D.R., C.G.S., T.D. and B.S. critically edited and revised the manuscript. M.M.B., K.D.R., P.J.M.S., N.B., J.H., R.T.A.M., C.G.S., T.D. and B.S. have approved the final version of the 
manuscript and agree to be accountable for all aspects of the work. All persons designated as authors qualify for authorship, and all those who qualify for authorship are listed.

\section{Funding}

BS was supported by a Kootstra Talent Fellowship by Maastricht University and by grants from the Netherlands Organisation for Scientific Research (Rubicon 452172006) and from the European Union's Horizon 2020 research and innovation program (No 793805).

\section{Competing interests}

The authors declare no competing interests.

\section{Additional information}

Supplementary Information The online version contains supplementary material available at https:/doi. org/10.1038/s41598-021-81151-5.

Correspondence and requests for materials should be addressed to B.S.

Reprints and permissions information is available at www.nature.com/reprints.

Publisher's note Springer Nature remains neutral with regard to jurisdictional claims in published maps and institutional affiliations.

(c) (1) Open Access This article is licensed under a Creative Commons Attribution 4.0 International License, which permits use, sharing, adaptation, distribution and reproduction in any medium or format, as long as you give appropriate credit to the original author(s) and the source, provide a link to the Creative Commons licence, and indicate if changes were made. The images or other third party material in this article are included in the article's Creative Commons licence, unless indicated otherwise in a credit line to the material. If material is not included in the article's Creative Commons licence and your intended use is not permitted by statutory regulation or exceeds the permitted use, you will need to obtain permission directly from the copyright holder. To view a copy of this licence, visit http://creativecommons.org/licenses/by/4.0/.

(C) The Author(s) 2021 